\section{ENGINEERING DATA TRANSMITTAL \\ $2 x_{2}$}

2. To: (Receiving Organization)

3. From: (Originating Organization)

Distribution

5. Proj./Prog./Dept./Div.:

Solid Waste Disposal 87430

$T$ Plant Engineering

6. Cog. Engr.:

W. John Geuther

8. Originator Remarks:

This Engineering Data Transmittal serves to route document WHC-SD-WM-ER-595, Iechnical Basis For Removal of 221-T Tunnel From Airborne Radiological Area status, for final review, approval, and release. The USQ Screening/Evaluation for this proposed change has been performed under USQ \# T-96-074.

\section{Receiver Remarks:

$$
\text { Desigu Baseline Doruinent }
$$$$
\text { nyes no }
$$

Page 1 of 1. EDT 605321
4. Related EDT No.:

$N / A$

7. Purchase Order No.:

N/A

9. Equip./Component No.:

$N / A$

10. System/Bldg_/Facility:

\section{1-T Tunne1}

12. Major Assm. Dwg. No.:

$$
\text { N/A }
$$

13. Permit/Permit Application No.: N/A

14. Required Response Date:

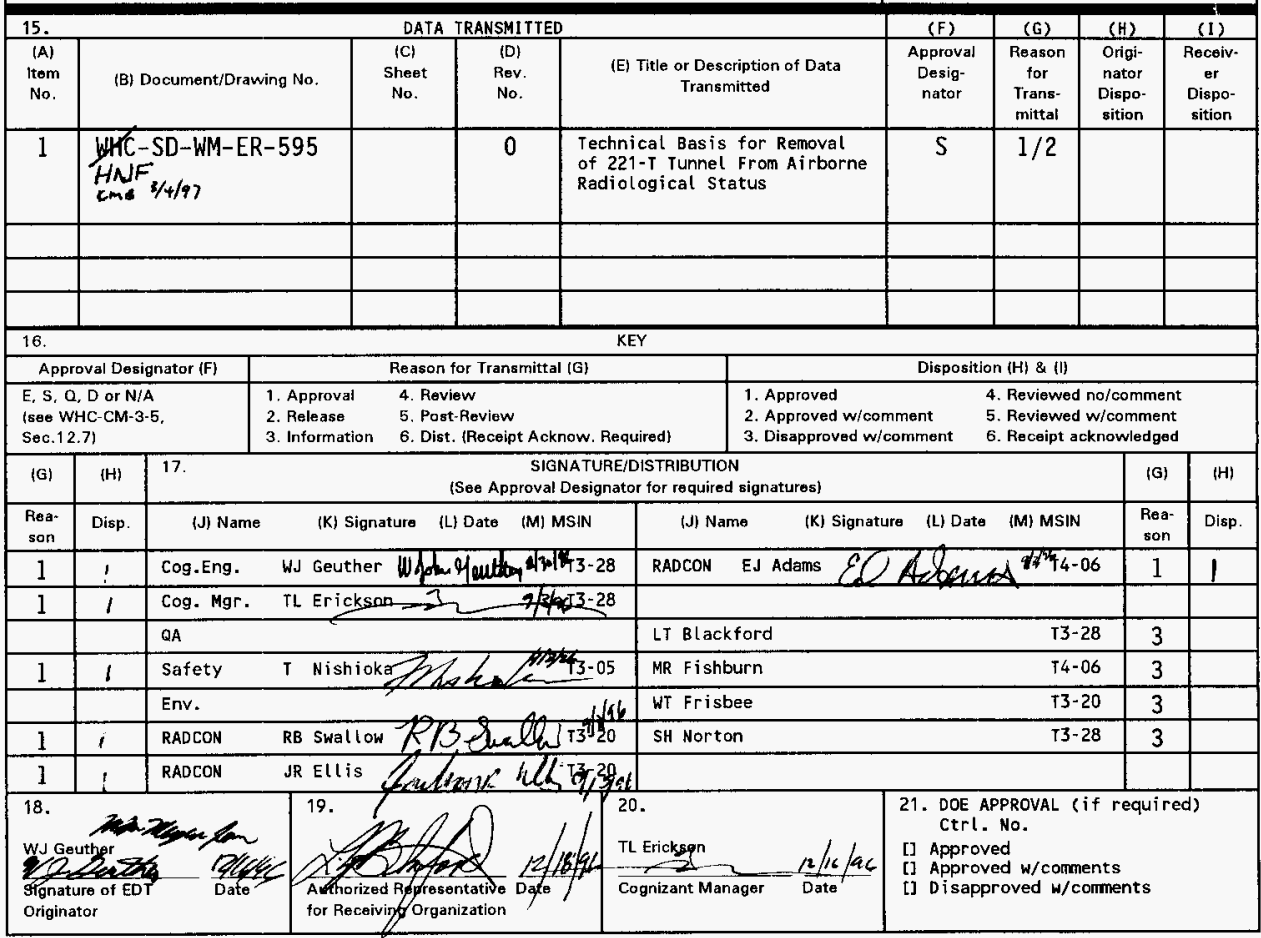




\title{
Technical Basis for Removal of 221-T Tunnel from Airborne Radiological Area Status
}

\author{
Jackson R. Ellis
}

Westinghouse Hanford Company, Richland, WA 99352

U.S. Department of Energy Contract DE-AC06-87RL10930

$\begin{array}{lll}\text { EDT/ECN: } & \text { EDT } 605321 & \text { UC: } 607 \\ \text { Org Code: } & 87 A 10 & \text { Charge Code: AA787 A32A3/4/77 } \\ \text { B\&R Code: } & \text { EW3130020 } & \text { Tota1 Pages: Jo } 4 / \text { Ras } 3 / 47\end{array}$

Key Words: radiological, airborne, respiratory, protection, tunnel, exposure, contamination

\section{Abstract:}

This document provides the technical basis for removal of the 221-T Tunnel from airborne radiological control. T Plant Radiological Control has evaluated air sampling data and engineering controls, and determined the necessary administrative controls to make this transition. With these administrative controls (specified within document) in place, the tunnel can be removed from Airborne Radioactive Area status. The removal of the tunnel from airborne status will allow work to be performed within the tunnel under controlled conditions, as outlined in this technical basis, without the use of respiratory protection equipment.

TRADEMARK DISCLAIMER. Reference herein to any specific commercial product, process, or service by trade name, trademark, manufacturer, or otherwise, does not necessarity constitute or imply its endorsement, recommendation, or favoring by the United States Government or any agency thereof or its contractors or subcontractors.

Printed in the United States of America. To obtain copies of this document, contact: WHC/BCS Document Control Services, P.0. Box 1970, Mailstop H6-08, Richland WA 99352, Phone (509) 372-2420; Fax (509) 376-4989.
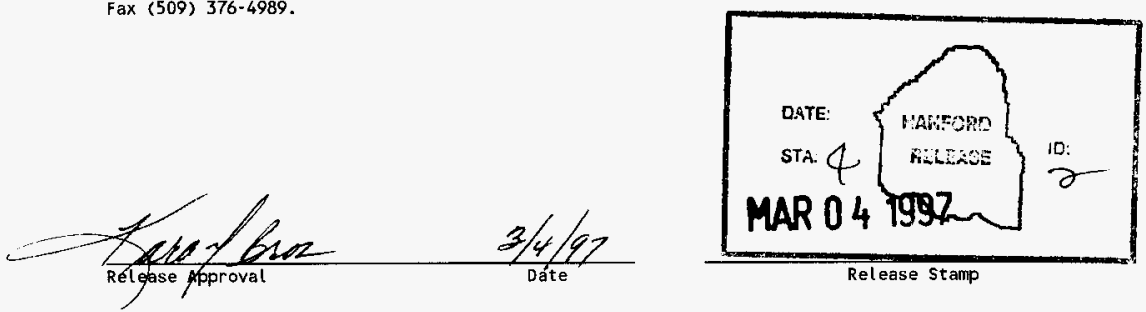

Approved for Public Release 


\section{TECHNICAL BASIS FOR REMOVAL OF 221-T TUNNEL FROM AIRBORNE RADIOLOGICAL AREA STATUS HNF-SD-WM-ER-595}

\section{SUMMARY}

T Plant Radiological Control has evaluated the 221-T Tunnel for removal from airborne radiological control. The removal of the tunnel from airborne status will allow work in the tunnel under controlled conditions, as outlined in this technical basis, to be performed without the use of respiratory protection equipment. T Plant Radiological Control has evaluated air sampling data and engineering controls, and determined the necessary administrative controls to make this transition. With these controls in place, the tunnel can function as a non-airborne radioactive area.

\section{HISTORY}

The 221-T Railroad Tunnel is an access route to allow railcars an avenue to the 221-T Canyon. The tunnel is located at the North end of the 221-T Canyon and is situated at a right angle to the canyon at a level below the canyon. The roof of the tunnel contains a $28^{\prime}$ by $16^{\prime}$ opening to the floor of the canyon.

The Hanford Site Radiological Control Manual (HSRCM-1), Table 2-4 states the criteria for posting an Airborne Radioactivity Area is $>10 \%$ Derived Air concentration (DAC). The downgrading of radiological controls for the tunnel can be accomplished due to its history of remaining below airborne limits while work was being performed in the canyon that caused airborne levels in excess of these limits. The attached graph (Attachment 1) contains air samples from the weeks of $4 / 25 / 95$ through $4 / 10 / 96$ for the continuous air monitor (CAM) that is located in the tunnel. The graphs demonstrate that the tunnel air did not exceed $10 \%$ of the DAC for alpha or beta airborne contamination. Airborne radioactive sample data (data analyzed from 1/96 to 4/96) demonstrates that the canyon air has exceeded $10 \%$ of the DAC for alpha or beta airborne contamination concentration while the tunnel has remained below these levels (Attachment 2).

\section{REQUIREMENTS}

HSRCM-1, Article 136 states, "In order to minimize internal exposures, managers should take deliberate actions to control contamination at the source and reduce Airborne Radioactivity, Contamination and High Contamination Areas. Work should be planned to avoid the routine use of respiratory protection devices." The HSRCM-1 also gives the following direction for control of internal exposure to radiological workers:

\section{Minimization of Internal Exposure}


The minimization and control of internal exposure as discussed in Article 136 should be conducted in accordance with the following hierarchy of controls:

1. Engineering controls, including containment of radioactive material at the source wherever practicable, should be the primary method of minimizing airborne radioactivity and internal exposure to workers.

2. Administrative controls, including access restrictions and the use of specific work practices designed to minimize airborne contamination, should be used as the secondary method to minimize worker internal exposure.

3. When engineering and administrative controls have been applied and the potential for airborne radioactivity still exists, respiratory protection should be used to limit internal exposures. Use of respiratory protection should be considered under the following conditions:
a. Entry into posted Airborne Radioactivity Areas
b. During breach of contaminated systems or components
c. Work in areas or on equipment with removable contamination levels greater than 100 times the values in Table 2-2
d. During work on contaminated or activated surfaces with the potential to generate airborne radioactivity.

In an effort to optimize the efficiency of work in the T Plant 221-T Tunnel, and to comply with the above requirements, T Plant management has initiated a plan to downpost the Tunnel from airborne radiological control status. Steps have been taken to characterize the contamination levels in the Tunnel, evaluate the engineering controls already in place, and update administrative controls for entry and work in the Tunnel.

\section{CHARACTERIZATION OF TUNNEL}

Contamination surveys were performed in the tunnel and the survey reports (Attachment 3) show that removable contamination levels are not above 100 times the values of HSRCM-1, Table 2-2 (requirement from HSRCM-1 316.3).

\section{ENGINEERING CONTROLS}


HNF-SD-WM-ER-595, Rev, 0

Page 3 of 4

The ventilation system of the canyon pulls outside air through the tunnel from around its doors, and into the canyon through the $28^{\prime}$ by $16^{\prime}$ opening.

Internal Memo, 33541-96-RBS-002, AIR FLOW PATTERNS IN THE 221-T TUNNEL

(Attachment 4), discusses the use of ventilation as a barrier against airborne contamination from the canyon entering the tunnel. The air flow study was

repeated with the railcar door completely opened and with a railcar present on May 30, 1996, and the test showed that the air flow was from the tunnel to the canyon in all cases. The latter test also demonstrated that the drain in the floor of the tunnel did not affect the air flow.

The roof exhaust fan for the canyon is isolated and is no longer functional as an airflow/emission point. Air flow with this system operating was not considered for this downposting. Supply fans in the canyon are usually not operated. Air flow studies were not conducted with these fans operating. The tunnel will be controlled as an airborne area if these fans are operating until the air flow study can be repeated with them turned on.

The activities that caused the air concentration in the canyon to exceed $10 \%$ DAC in 1996 (see Attachment 2) were cutting of contaminated ductwork at Section 10 and auger decontamination activities at Section 15. These activities are conducted $>300$ and $>500$ feet away from the tunnel entry area, respectively. The auger decontamination activities are usually performed daily for approximately 4 hours each day. Air sampling is performed at the location of auger decontamination.

\section{ADMINISTRATIVE CONTROLS}

The engineering controls are supplemented with administrative controls described in T Plant Plant Operating Procedures (POP), Radiological Work Permits (RWP), work control reviews, and Radiological Control routine tasks. Administrative controls to be utilized include:

Access under normal conditions from the canyon to the tunnel and vice versa will not be allowed. Special considerations will be made for individual jobs where these accesses are needed. These are controlled with radiological postings and RWPS.

Activities in the tunnel where respiratory protection is utilized will require the entire tunnel will be posted as an Airborne Radioactivity Area. These are controlled with radiological postings and RWPs. Downposting survey plans will be used to return the tunnel to its normal status.

Once the tunnel is downposted, routine surveys will be performed to ensure that removable contamination is not present that could cause airborne contamination. This is implemented by in routine T-W010, Contamination and Dose Rate Survey of Tunnel.

The area in the canyon around the tunnel entry will be routinely surveyed for removable contamination to ensure contaminated debris from 
the canyon does not fall into the tunnel. This is covered in routine T-WO1l, Contamination and Dose Rate Survey of Canyon Craneway Opening and PWR Pool Area.

Controls will be used to ensure that the crane hook is not lowered into the tunnel with removable contamination levels greater than 100 times the values of Table 2-2 (HSRCM-1) of contamination on it. This is controlled by the Crane Procedure D0-021-029, which contains a step that requires a contamination survey of the hook to ensure levels as described on the RWP are not exceeded.

Controls will be used to ensure that non-routine jobs that could potentially cause increased levels of airborne contamination (e.g. movement of cell block covers on highly contaminated cells) are not performed in the canyon North of the Section 10 ( -300 feet from tunnel entrance) with the tunnel "off mask." Work control and RWPs will control these individually as they are performed.

Controls will be used to ensure that the tunnel is not off mask if failure of the canyon ventilation system occurs. Evacuation of the tunnel is required in the event of a low pressure alarm as described in ARP-D-463-00002 Respond to 221-T, 221-T52, \& 291-T Alarms. Returning to normal operation condition will occur as part of emergency recovery depending on circumstances.

Work place air sampling for the tunnel will include continuous air monitoring to alert personnel for increases in the airborne concentration. An air sampler will be used to obtain a record air sample. Grab samples will taken for specific jobs to determine airborne concentrations during work.

Canyon ventilation supply fans will be locked and tagged out of service to prevent operation while personnel without respiratory protection are in the tunnel.

\section{CONCLUSION}

T P1ant Radiological Control has evaluated the 221-T Tunnel for removal from airborne radiological control. T Plant has implemented the necessary controls described in this Technical Basis. With these controls in place, the tunnel can function as a non-airborne radioactive area. 


\section{TRACKING REPORT FOR EDP-T}

OO 1 TUNNEL

(The Dates are projected TIME OFF Dates of the air samples)

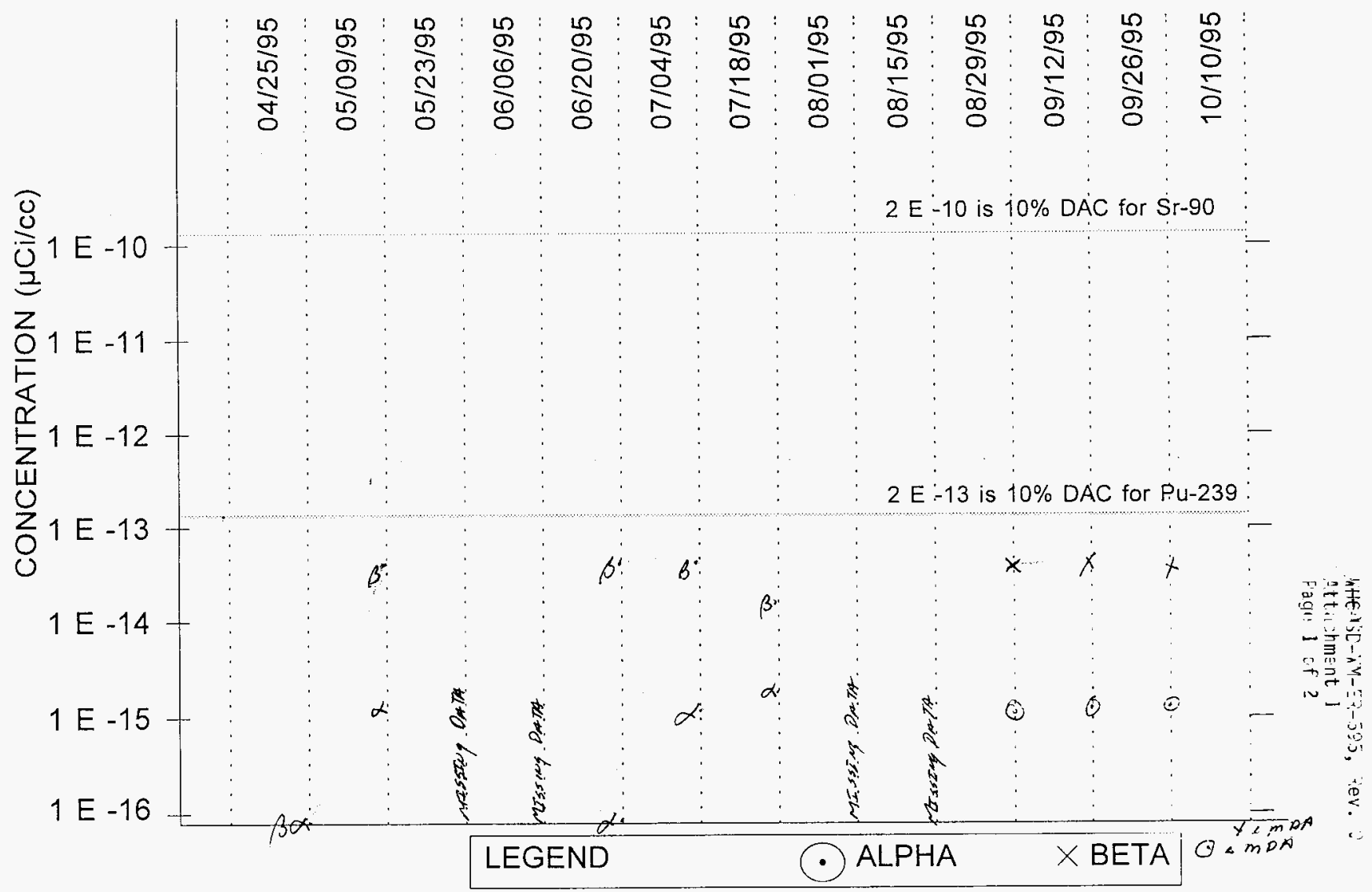




\section{TRACKING REPURT FOR EDP-T ool}

(The Dates are projected TIME OFF Dates of the air samples)

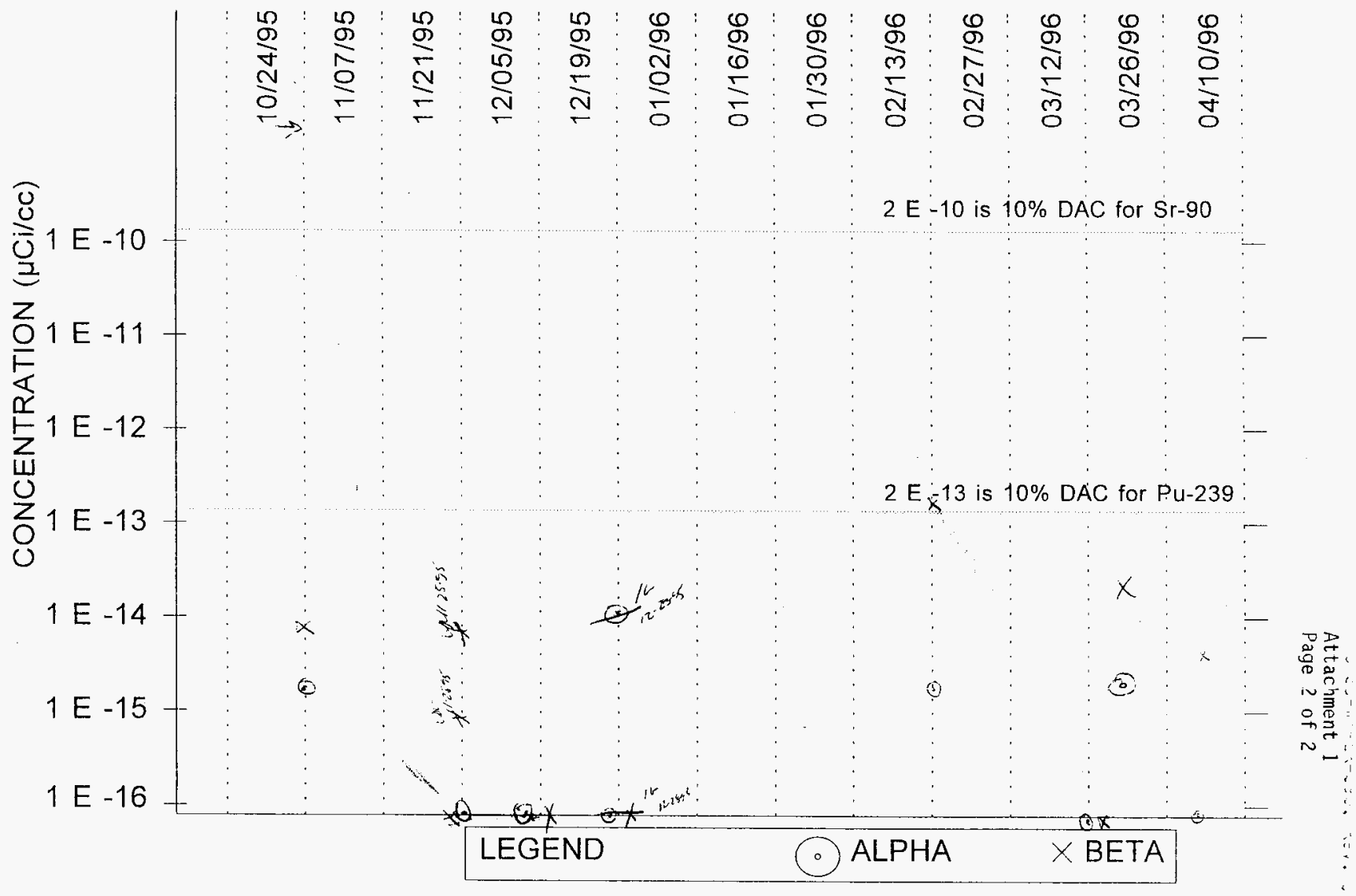


HNF-WHC-SD-WM-ER-595, Rev. 0 Attachment 2

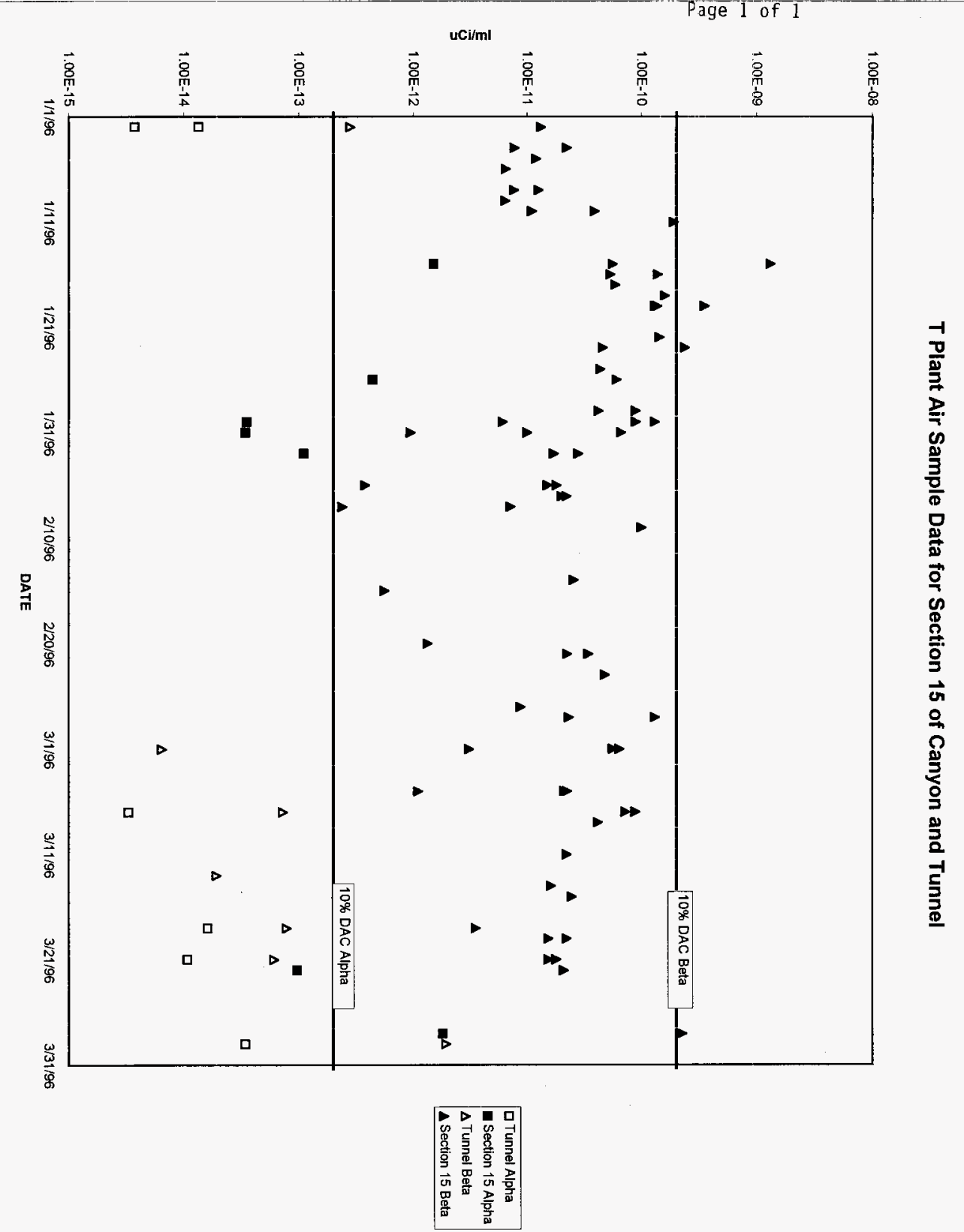




\section{Westinghouse Hanford Company - Radiological Control RADIOLOGICAL SURVEY REPORT}

\begin{tabular}{|c|c|c|c|c|c|c|}
\hline $6 / 19 / 96$ & $\begin{array}{l}\text { Time } \\
0900-153\end{array}$ & $\begin{array}{r}\text { RWP No.(s) } \\
T-115\end{array}$ & Page & 1 & of & 2 \\
\hline $\begin{array}{l}\text { Aroo/Aldg./Room/Lo } \\
2 \omega / 221-T\end{array}$ & $\begin{array}{l}\text { CodeI } \\
\text { NNEC / ROLe }\end{array}$ & LP AOar & & & & $\frac{1}{1}$ \\
\hline
\end{tabular}

Job Description INUESTIGATUE SURVEY Purpose of Survey (check appropriate box[esi):

of TuNNEL FLOOR WACCS, AND Contamination Incident: $\square$ skin, $\square$ Clothing. $\square$ spil

Alarm Response: $\square$ CAM, $\square$ ARM(RAM). $\square$ PSD
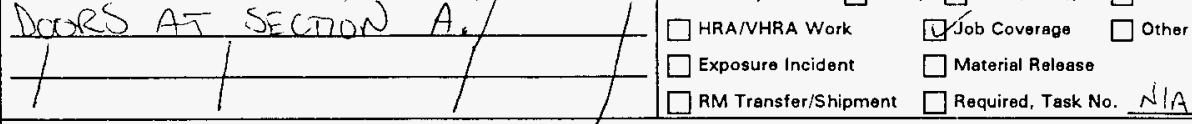

$\square$ Exposure Incident $\square$ Material Release

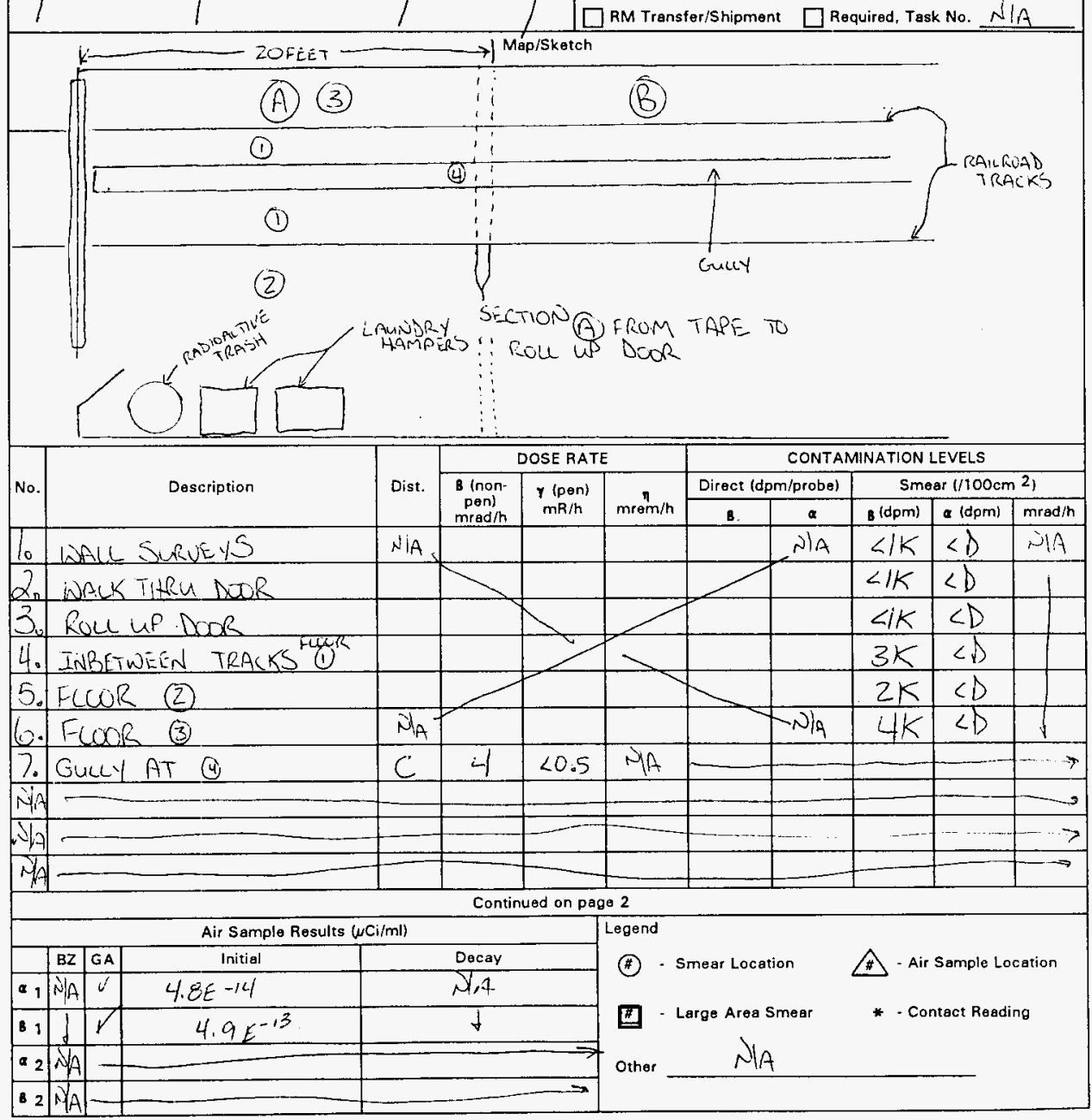




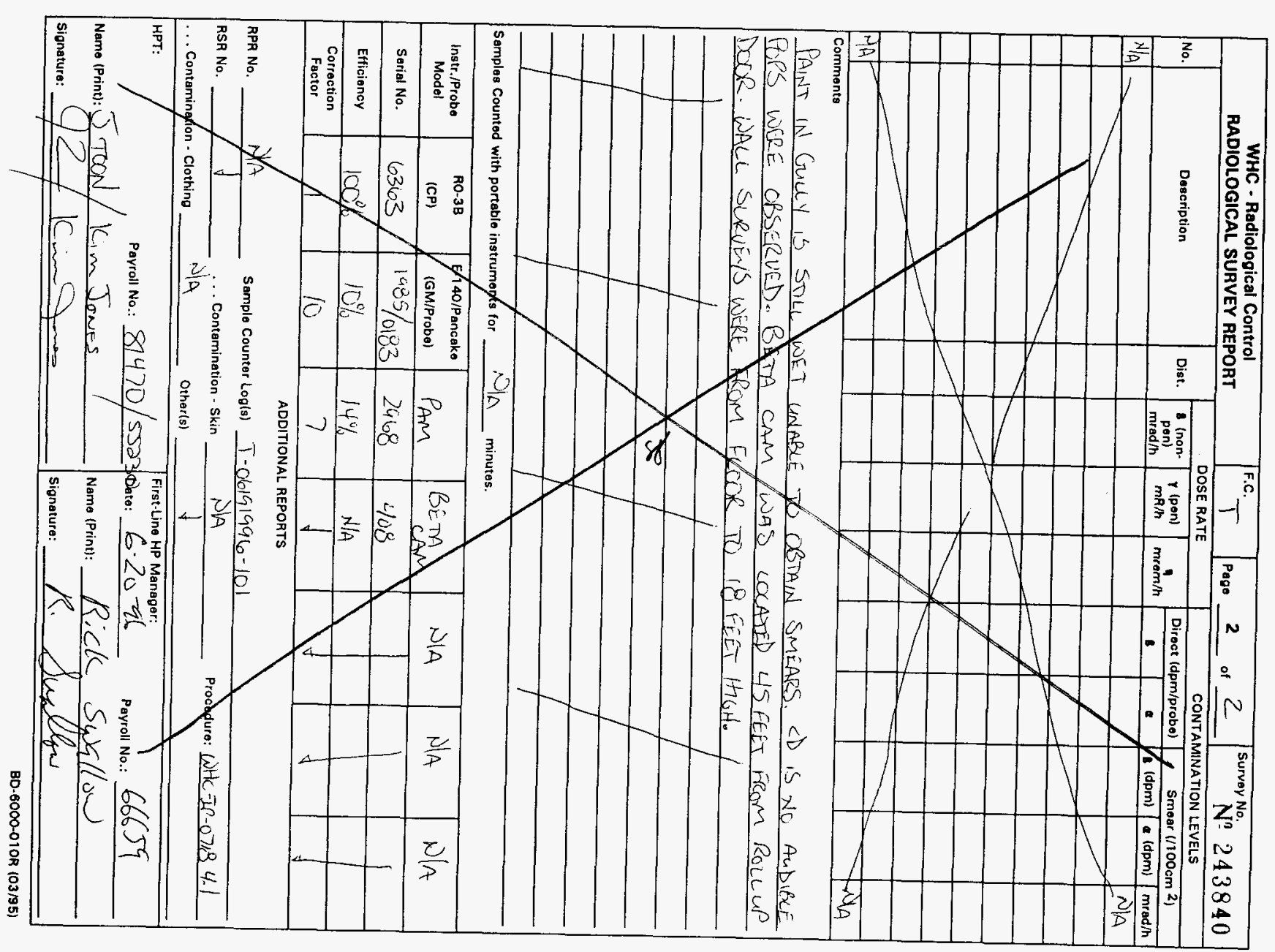




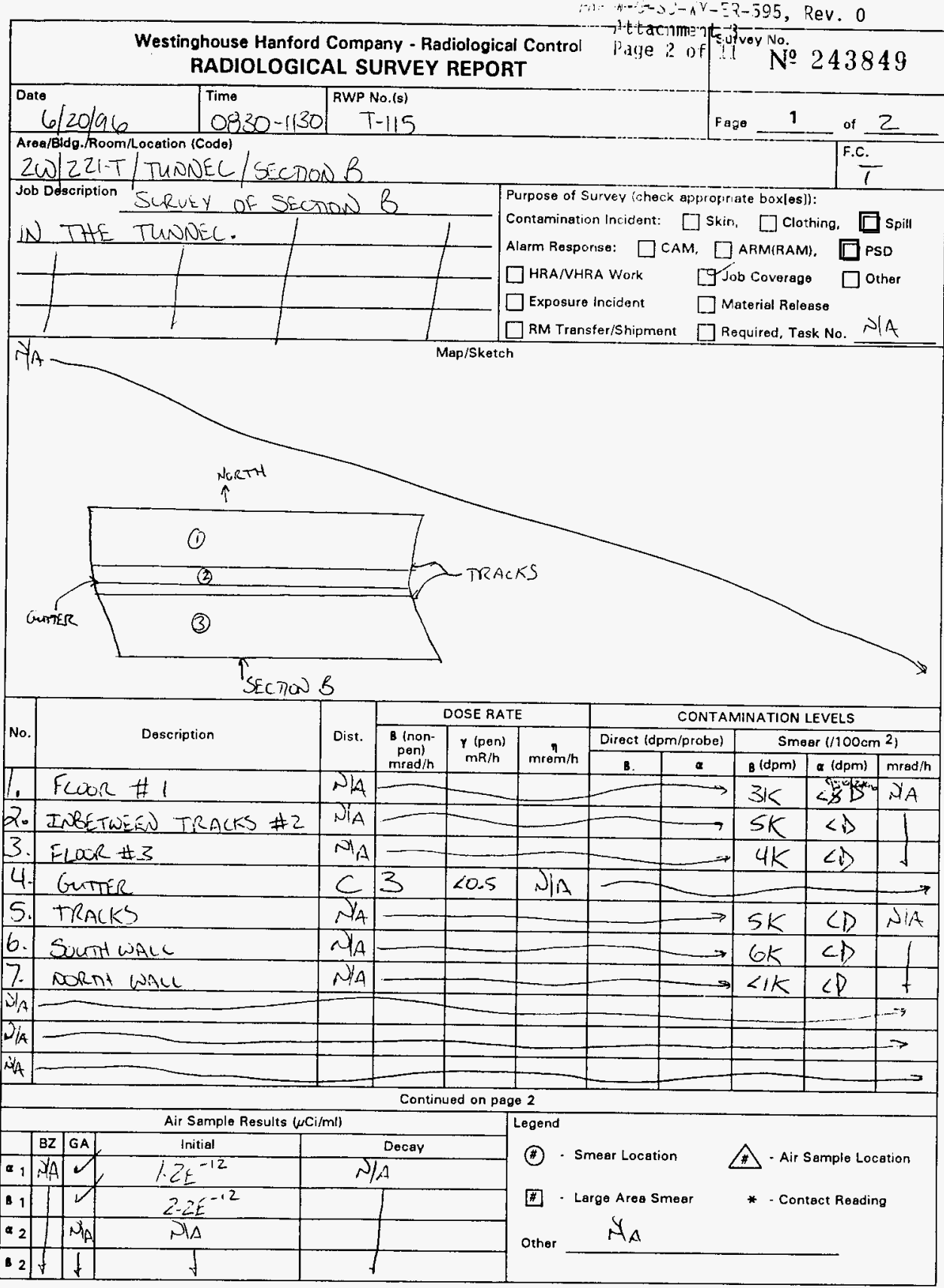




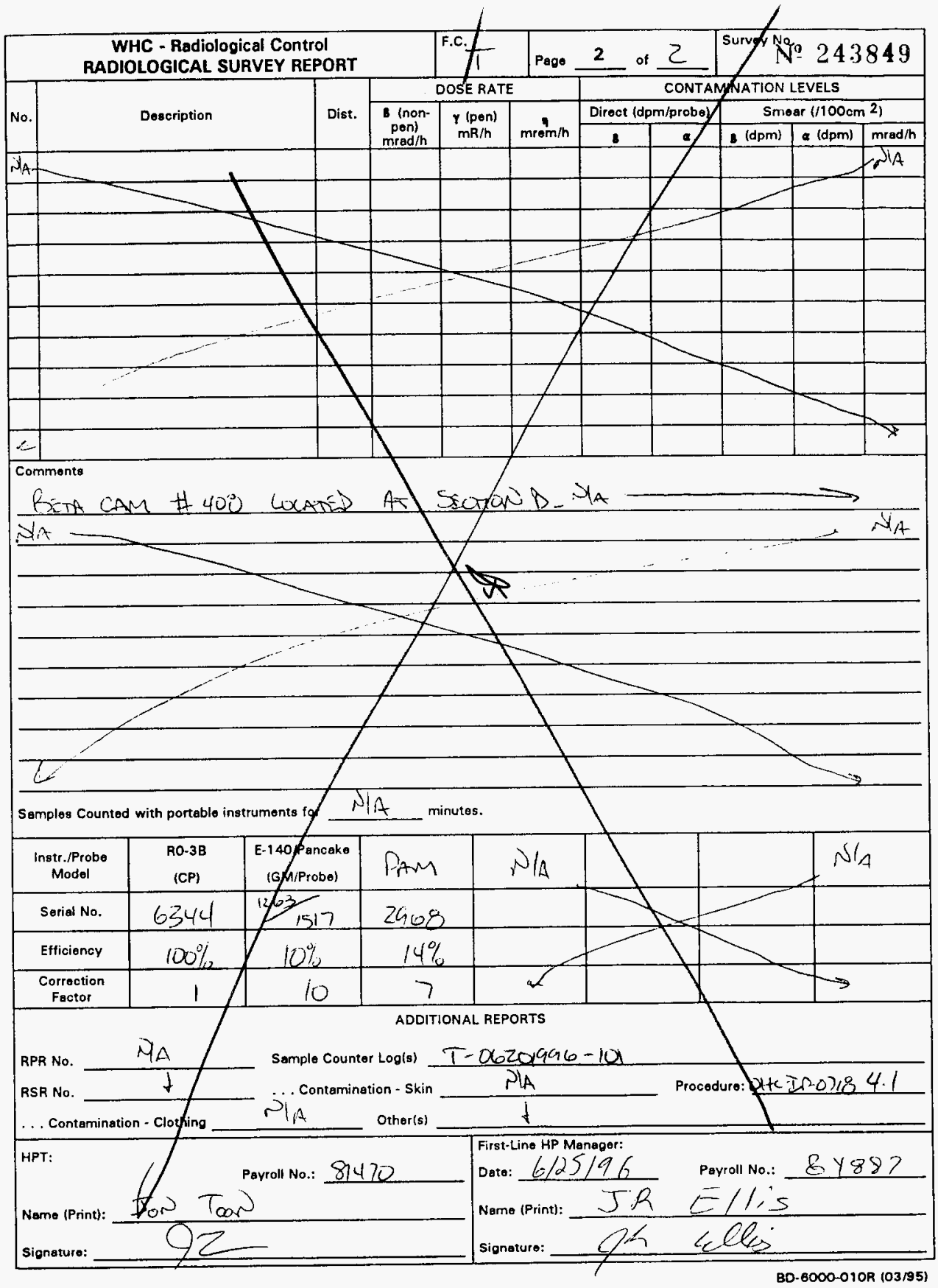


Westinghouse Hanford Company - Radiological Control

RADIOLOGICAL SURVEY REPORT

\section{Date}

$6-20.96$

RWP No.(s)

$T-230$

Acosalardo-/Room/Location (Code) $/ 2717 / T u n n e / / 08$

Job Description Survey of tunnel for clean up and decoutanination
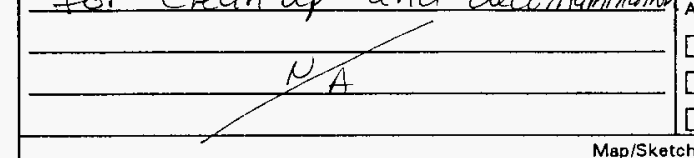

№ 243852

Pago 1 of 23
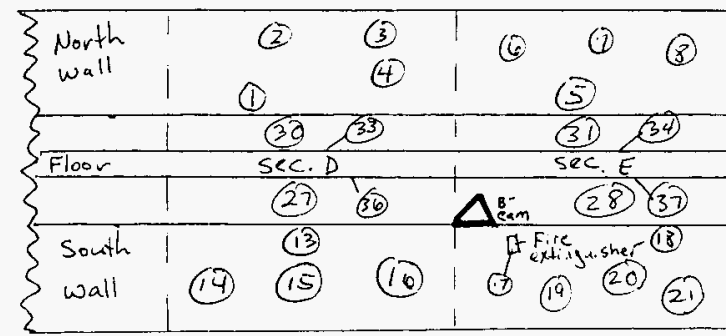

(10)

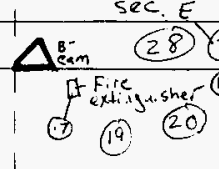

(37)

Purpose of Survey (check appropriate box[es]):

Contamination Incident: $\square$ skin, $\square$ Clothing. $\square$ spill

Alarm Response:

CAM, $\square$ ARM(RAM).

$\square$ PSD

$\square$ HRA/VHRA Work $\square$ Jab Coverage $\square$ Other

$\square$ Exposure Incident $\square$ Material Release

RM Transfer/Shipment $\square$ Required, Task No.

$$
\text { cangon Tunnel w } \frac{\int_{s}^{N} E}{s}
$$

\begin{tabular}{|c|c|c|c|c|c|c|c|c|c|c|c|c|}
\hline \multirow{3}{*}{ No. } & \multirow{3}{*}{\multicolumn{3}{|c|}{ Description }} & \multirow{4}{*}{$\begin{array}{l}\text { Dist. } \\
F / A\end{array}$} & \multicolumn{3}{|c|}{ DOSE RATE } & \multicolumn{5}{|c|}{ CONTAMINATION LEVELS } \\
\hline & & & & & \multirow{2}{*}{$\begin{array}{l}8 \text { (non- } \\
\text { pen) } \\
\text { mrad/h }\end{array}$} & \multirow{2}{*}{$\begin{array}{c}Y \text { (pen) } \\
\mathrm{mR} / \mathrm{h}\end{array}$} & \multirow{2}{*}{$\mathrm{mrem} / \mathrm{h}$} & \multicolumn{2}{|c|}{ Direct (dpm/probe) } & \multicolumn{3}{|c|}{ Smear $\left(/ 100 \mathrm{~cm}^{2}\right)$} \\
\hline & & & & & & & & B. & $\alpha$ & $B(\mathrm{dpm})$ & $a(\mathrm{dpm})$ & $\mathrm{mrad} / \mathrm{h}$ \\
\hline 1 & \multicolumn{3}{|c|}{ N. Wall Sec D } & & & & & & $1 / 4$ & $2 K$ & $<P$ & $N / A$ \\
\hline 2 & \multicolumn{3}{|c|}{ N. wall Sec. D } & & & & & & & $5 K$ & $\angle D$ & 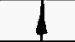 \\
\hline 3 & \multicolumn{3}{|c|}{ N. Wall Sec. D } & & & & & & & $4 k$ & $\angle P$ & \\
\hline 4 & \multicolumn{3}{|c|}{ N. Wall Sec D } & & & & & & & $5 K$ & $\angle D$ & \\
\hline 5 & \multicolumn{3}{|c|}{ N. Wall Sec. E } & & & & & & & $3 K$ & $\angle D$ & \\
\hline$k$ & \multicolumn{3}{|c|}{ N. Wall Sec E } & & & & & & & $3 K$ & $\leq D$ & \\
\hline 7 & \multicolumn{3}{|c|}{ N. Wall Sec. E } & & & & & & & $5 K$ & $\angle D$ & \\
\hline 8 & \multicolumn{3}{|c|}{ N. wall Sec E } & & & & & & & $7 K$ & $\angle D$ & \\
\hline 9 & \multicolumn{3}{|c|}{ N. Wall sec F } & & & & & & & $3 K$ & $\angle D$ & 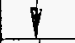 \\
\hline 10 & \multicolumn{3}{|c|}{ N. nall Sec. F } & $N / A$ & & & & & $N / A$ & $-7 k$ & $\angle 1$ & $\pi / A$ \\
\hline \multicolumn{13}{|c|}{ Continued on page 2} \\
\hline & \multicolumn{6}{|c|}{ Air Sample Results $(\mu \mathrm{Ci} / \mathrm{ml})$} & \multirow{2}{*}{\multicolumn{3}{|c|}{$\begin{array}{l}\text { Legend } \\
\text { \# - Smear Lo }\end{array}$}} & \multirow{2}{*}{\multicolumn{3}{|c|}{ A. Air Sample Location }} \\
\hline & Bz & $\mathrm{GA}$ & Initial & \multicolumn{3}{|c|}{ Decay } & & & & & & \\
\hline$\alpha_{1}$ & $\operatorname{con}$ & $X$ & $2.54 E-13$ & \multicolumn{3}{|c|}{$N A$} & \multirow{4}{*}{$\begin{array}{l}\text { D. } \\
\text { Other }\end{array}$} & \multicolumn{2}{|c|}{ Smear Lo } & \multirow{2}{*}{\multicolumn{3}{|c|}{ * - Contact Reading }} \\
\hline 81 & & $X$ & $3.06 E-12$ & \multicolumn{3}{|c|}{ NAt } & & \multicolumn{2}{|c|}{ Largo Area Smear } & & & \\
\hline$a_{2}$ & & & NA & \multicolumn{3}{|c|}{$N / 1$} & & \multicolumn{2}{|c|}{$N / A$} & & & \\
\hline$B_{2}$ & 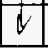 & & $N A$ & & $N A$ & & & & & & & \\
\hline
\end{tabular}


(S6/EO) y०10-0009-08

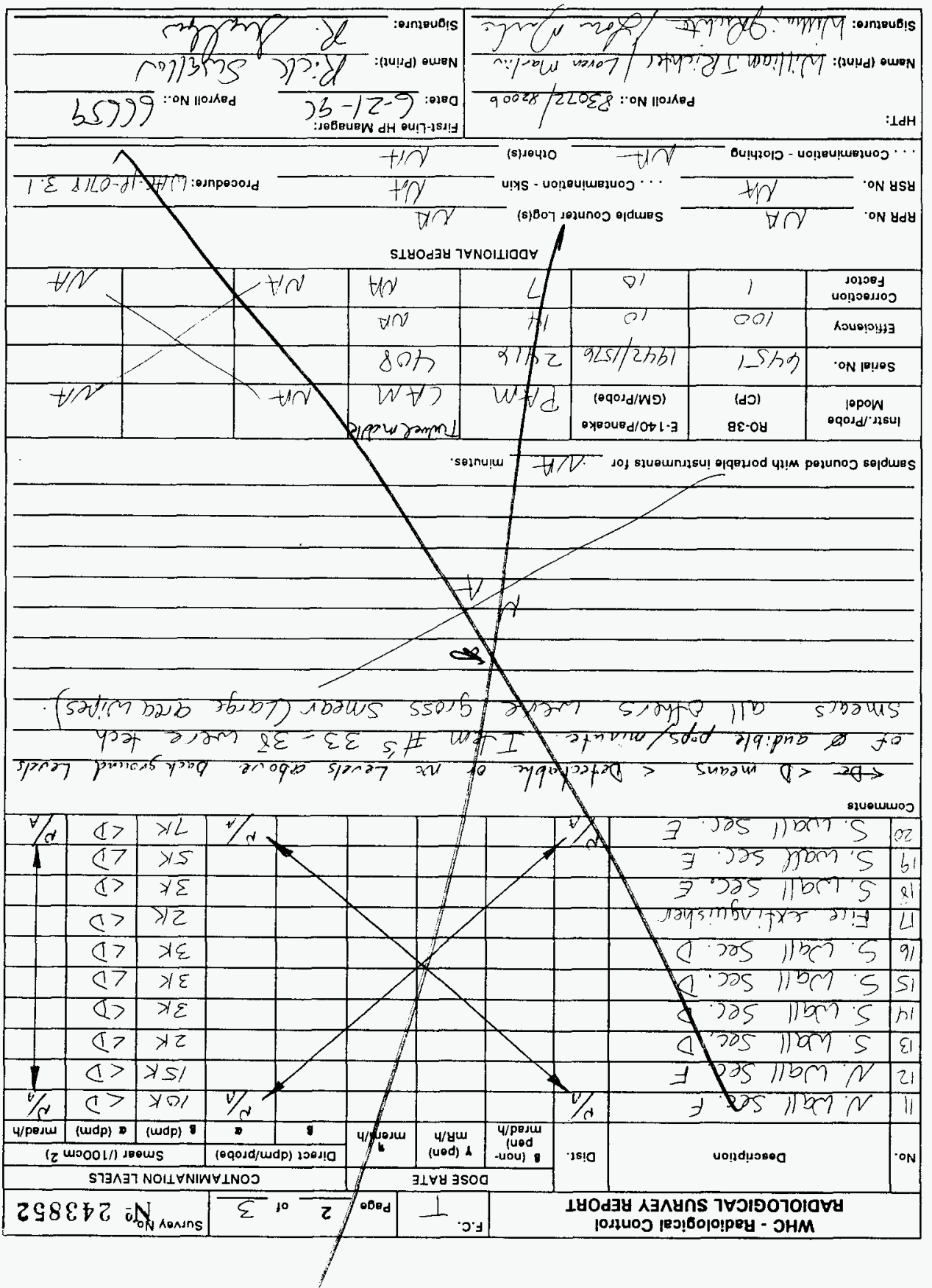




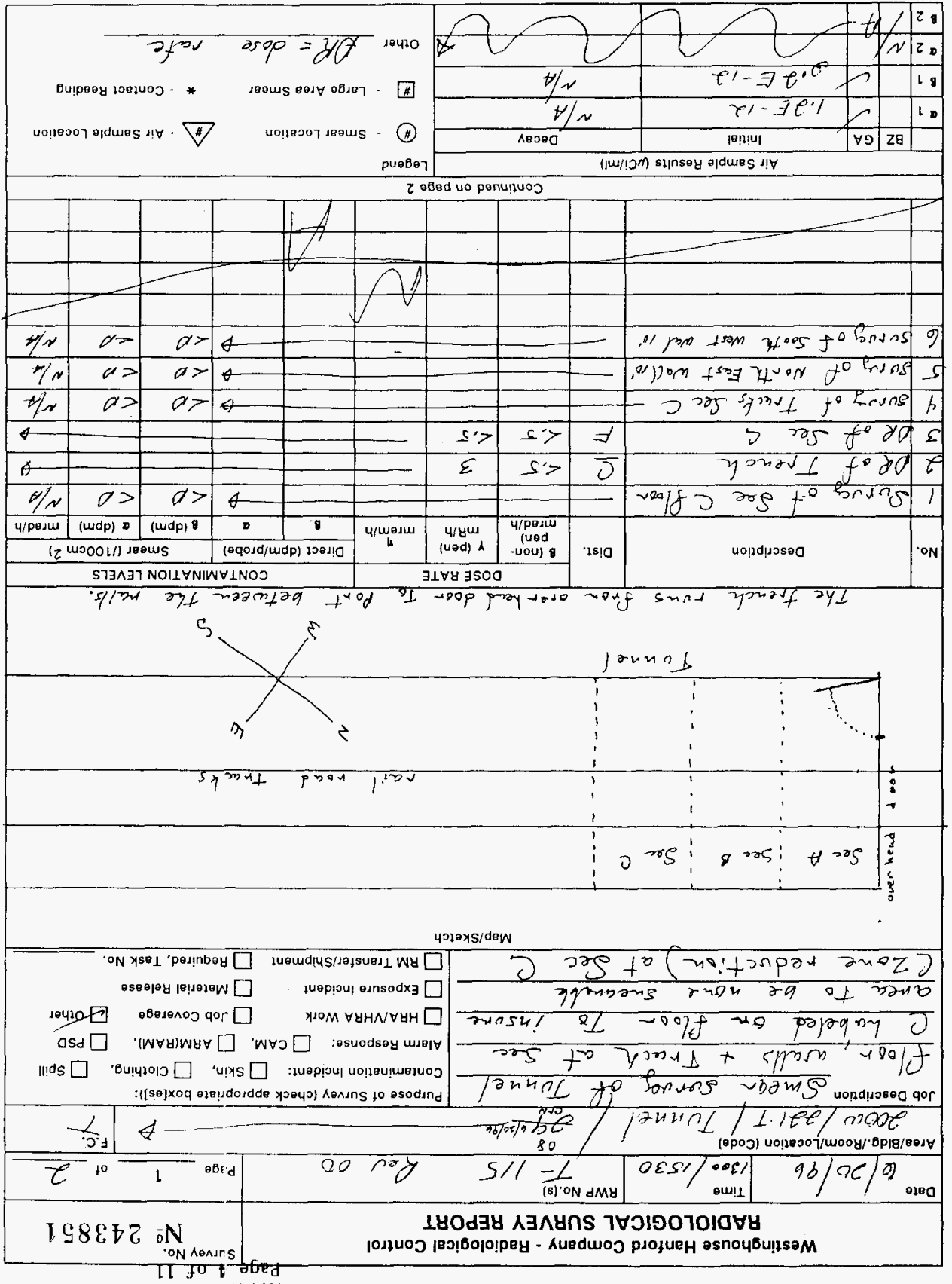

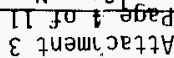




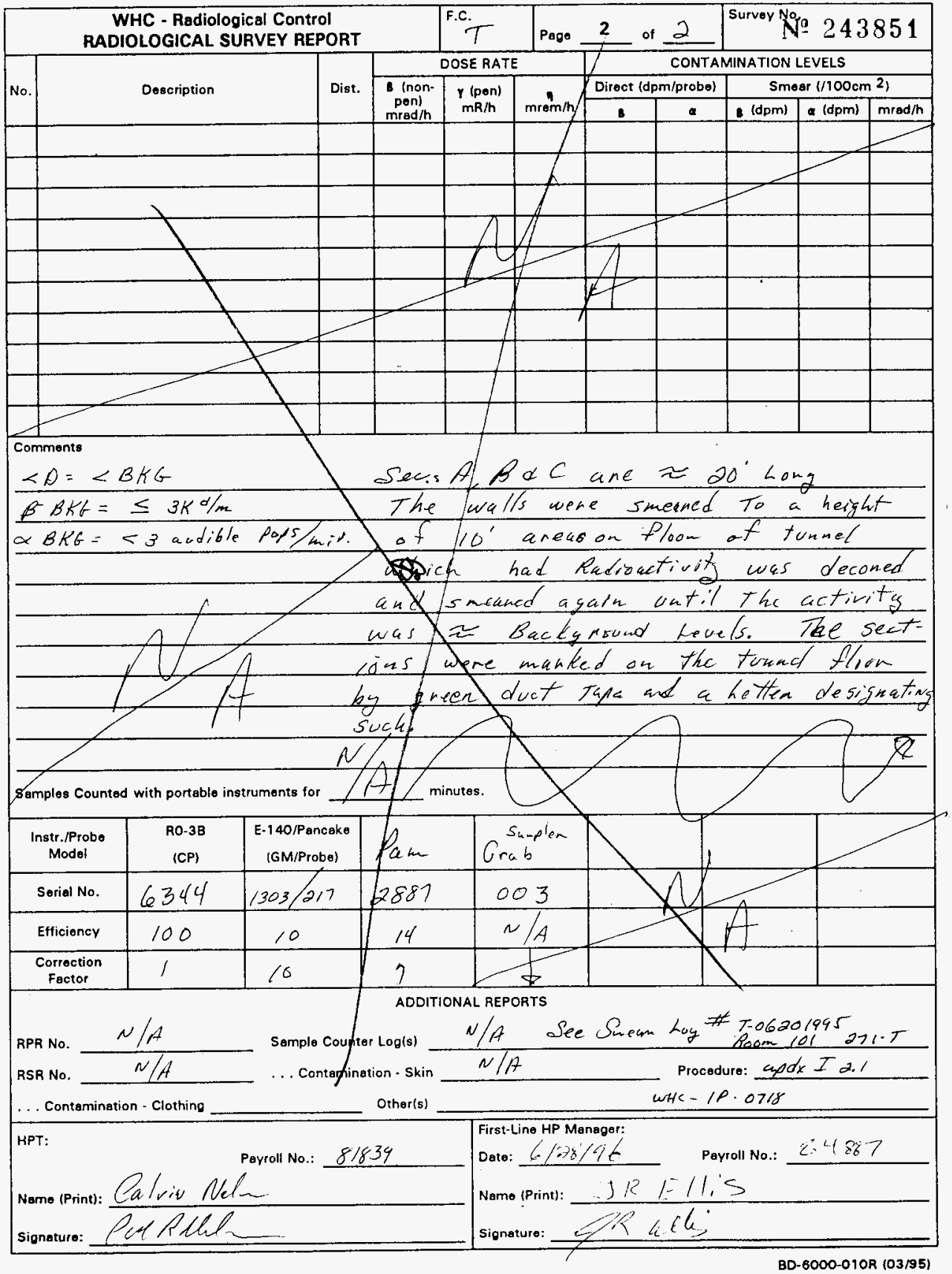


Attaciment 3

Page 5 of 11

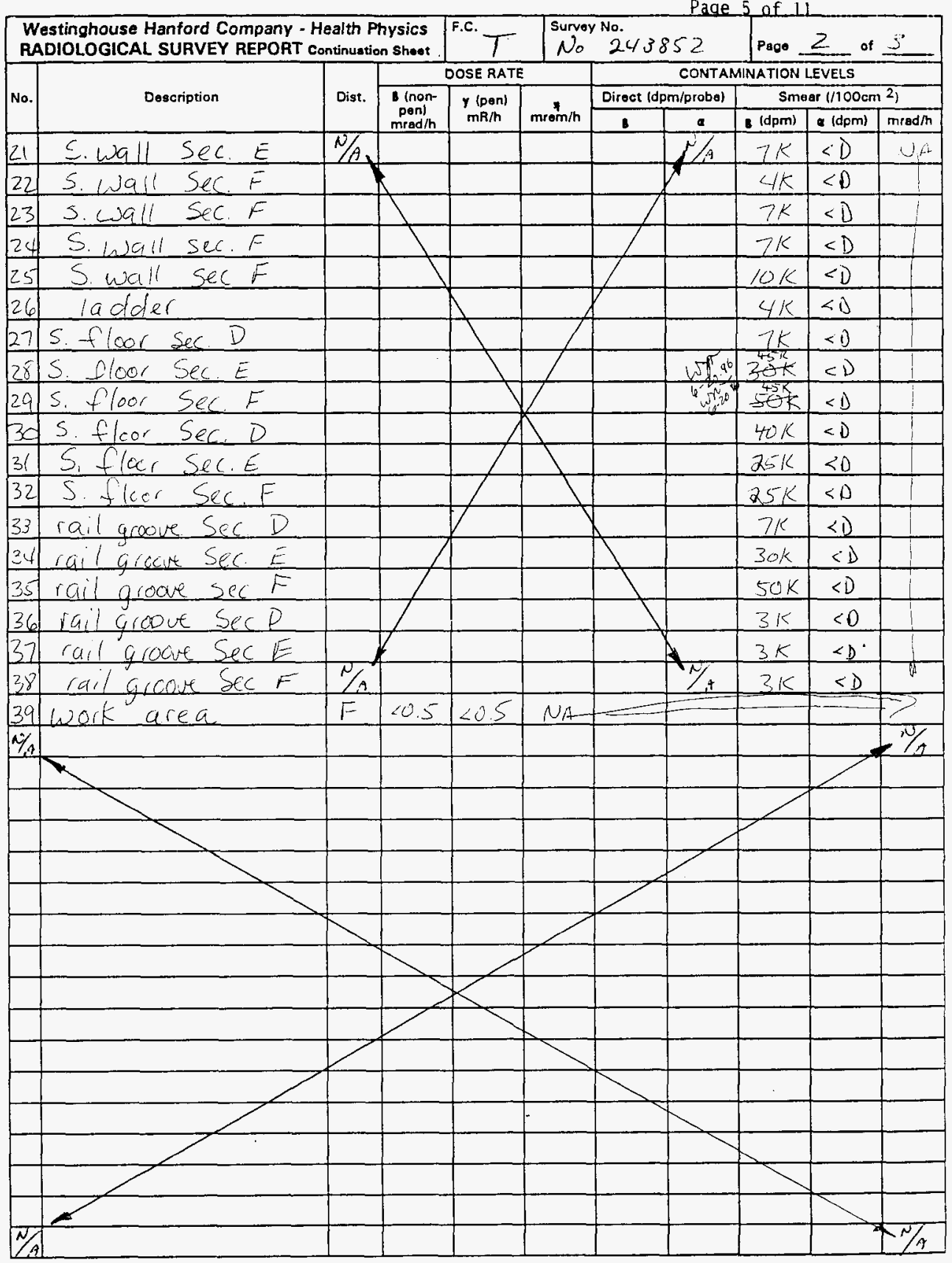




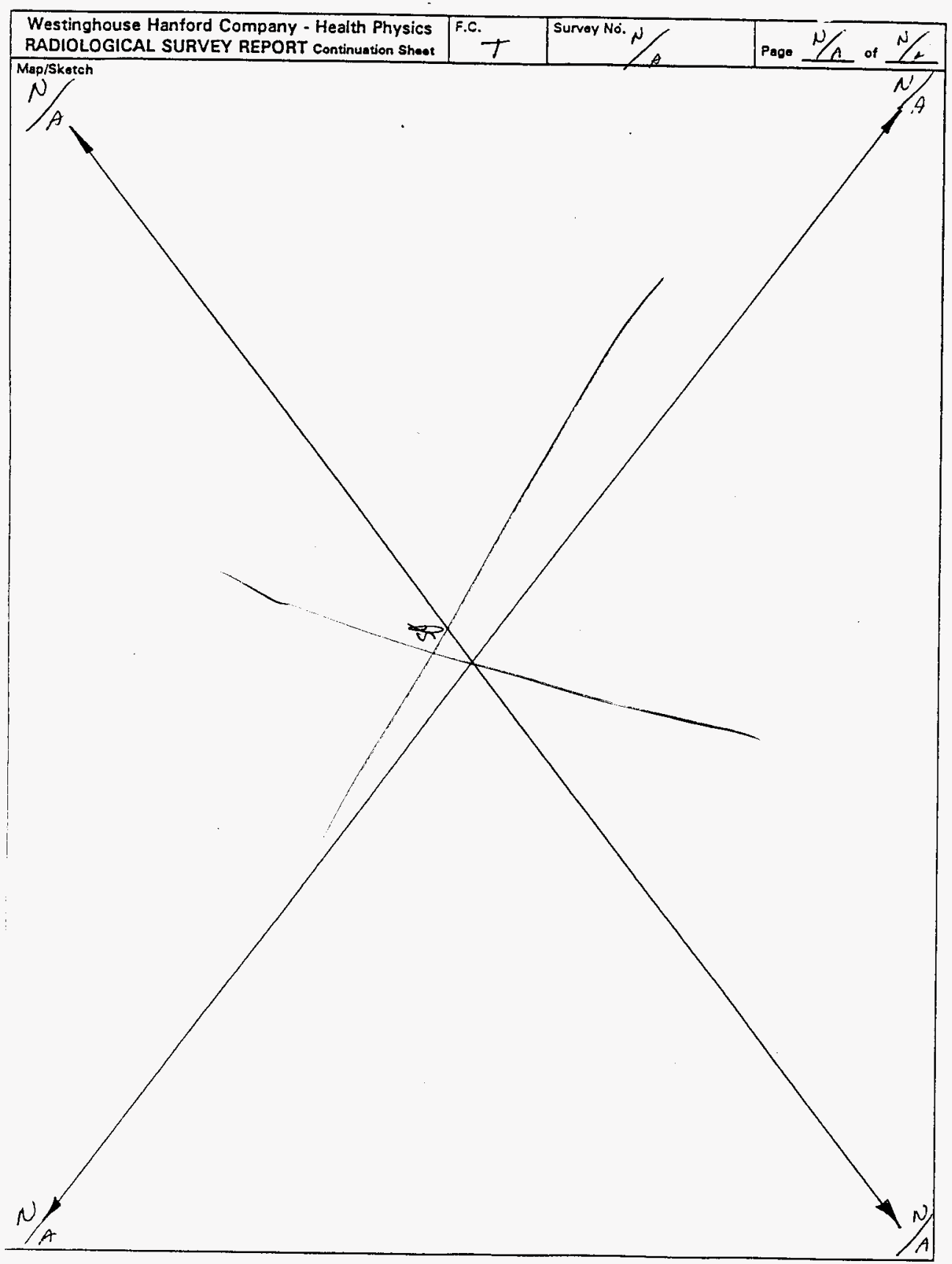


Westinghouse Hanford Company - Radiological Control RADIOLOGICAL SURVEY REPORT

\begin{tabular}{|c|c|c|}
\hline $\begin{array}{l}\text { Dato } \\
00-21.0 \% 0\end{array}$ & Time 285,1130 & $\begin{array}{r}\text { RWP No.(s) } \\
T-115\end{array}$ \\
\hline $\begin{array}{l}\text { Aroo/Bldg./Room } / 1 \\
20 x \times d 2\end{array}$ & Munal & \\
\hline
\end{tabular}

$\operatorname{sect} \cdot F$

pol1 U U door

$4-$

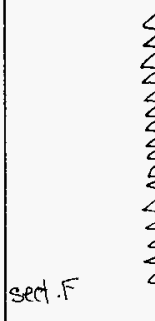

Map/Sketch

Northusall

Morth Rall

Rail Trench

Sooth RaN

op sooth wall

6.21 .56 sect. 6

\section{No 243854}

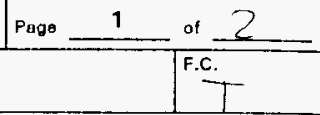

Purpose of Survey (check appropriate box[es]):

Contamination Incident: $\square$ skin, $\square$ clothing. $\square$ spill Alarm Response: $\square$ CAM. $\square$ ARM(RAM), $\square$ PSO

$\square$ HRA/VHRA Work $\square$ Job Coverage $\square$ Other

$\square$ Exposure Incident $\square$ Material Release

$\square$ RM Transfer/Shipment $\square$ Required, Task No.

Tunnd opening $\rightarrow$ to cahyon

\begin{tabular}{|c|c|c|c|c|c|c|c|c|c|c|c|c|}
\hline \multirow{3}{*}{ No. } & \multirow{3}{*}{\multicolumn{3}{|c|}{ Description }} & \multirow{3}{*}{ Dist. } & \multicolumn{3}{|c|}{ DOSERATE } & \multicolumn{5}{|c|}{ CONTAMINATION LEVELS } \\
\hline & & & & & \multirow{2}{*}{$\begin{array}{c}\text { Bnon- } \\
\text { pend } \\
\mathrm{mrad} / \mathrm{h}\end{array}$} & \multirow{2}{*}{$\begin{array}{c}Y \text { (pen) } \\
\mathrm{mR} / \mathrm{h}\end{array}$} & \multirow{2}{*}{$m n^{n} \operatorname{mon}$} & \multicolumn{2}{|c|}{ Direct (dpm/probe) } & \multicolumn{3}{|c|}{ Smear $\left(/ 100 \mathrm{~cm}^{2}\right)$} \\
\hline & & & & & & & & B. & $a$ & B (dpm) & a (dpm) & $\mathrm{mrad} / \mathrm{h}$ \\
\hline 1 & \multicolumn{3}{|c|}{ bross smar S. upper wall } & $\ldots$ & & & $m$ & &...$-+\ldots$ & 9,000 & $\angle b \times g$ & $\cdots$ \\
\hline 2 & \multicolumn{3}{|c|}{ gross smears. lawer wall } & - & & & & & - & 4,000 & $2 b<9$ & - \\
\hline 3 & \multicolumn{3}{|c|}{ bross smear N. Uoper wall } & $\ldots$ & & & & - & - & 4,000 & $20 \times 9$ & sh \\
\hline 4 & \multicolumn{3}{|c|}{ Gross smear $M$. lower wall } & $\ldots$ & & & & & $+\ldots$ & 5,000 & $20 \times 9$ & - \\
\hline 5 & \multicolumn{3}{|c|}{ aross smrar S Rail } & & & & & & - & 30,000 & Lbeg & $\rightarrow$ \\
\hline 6 & \multicolumn{3}{|c|}{ Gross smear N. Rail } & - & & & & & - & 7,000 & 2 bxa & - \\
\hline 7 & \multicolumn{3}{|c|}{ Gross smear S. Fioor betwon } & - & & & & & & $\ldots$ & & $=$ \\
\hline- & \multicolumn{3}{|c|}{ rail suall } & - & & & & & - & 10,000 & $\angle b \times a$ & - \\
\hline 8 & \multicolumn{3}{|c|}{ Gross smear inside vails } & & & & & & 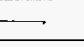 & 20,000 & $\angle$ bica & - \\
\hline & \multicolumn{3}{|c|}{$\begin{array}{l}\text { gross smear N floor between } \\
\text { rail zwall }\end{array}$} & - & & & & & $\ldots$ & 7,000 & cbrg & $\cdots$ \\
\hline & & & & & Contin & Led on pa & ge 2 & & & & & \\
\hline & \multicolumn{6}{|c|}{ Air Sample Results $(\mu \mathrm{Ci} / \mathrm{m} \|)$} & \multirow{3}{*}{$\begin{array}{l}\text { Legend } \\
\text { (\#) - }\end{array}$} & & \multirow{2}{*}{\multicolumn{3}{|c|}{ A Air Sample Location }} \\
\hline & $\mathrm{BZ}$ & $G A$ & Initial & \multicolumn{3}{|c|}{ Decay } & & & & & & \\
\hline$a_{1}$ & & & $75\{-13$ & 2 & & & & \multicolumn{2}{|c|}{ Smear Location } & \multirow{2}{*}{\multicolumn{3}{|c|}{ * Contact Reading }} \\
\hline 1 & $y$ & & $3.06-12$ & \multicolumn{3}{|c|}{ HAn } & \# & \multicolumn{2}{|c|}{ Large Area Smear } & & & \\
\hline 2 & - & 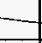 & & & & & Other & & $N \mid A$ & & & \\
\hline 82 & & & & & & & & & & & & \\
\hline
\end{tabular}




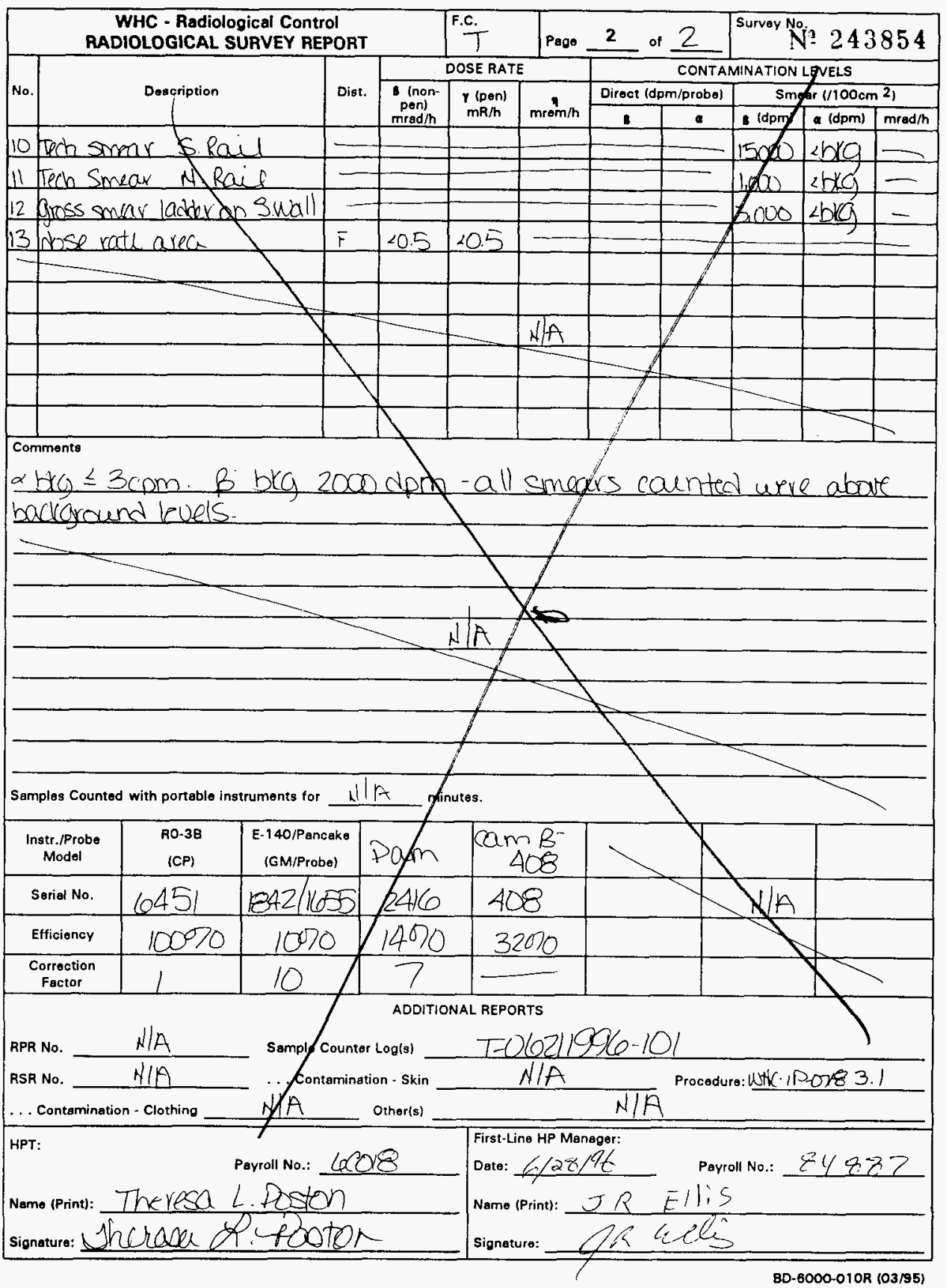


4 sections In the Tunvel

( $\sec 1 t, I, I$, and $k)$ Ans the

Stair well Fon the Tound

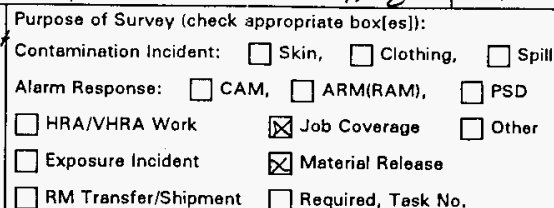
Flow to the CAt wyan Deck.
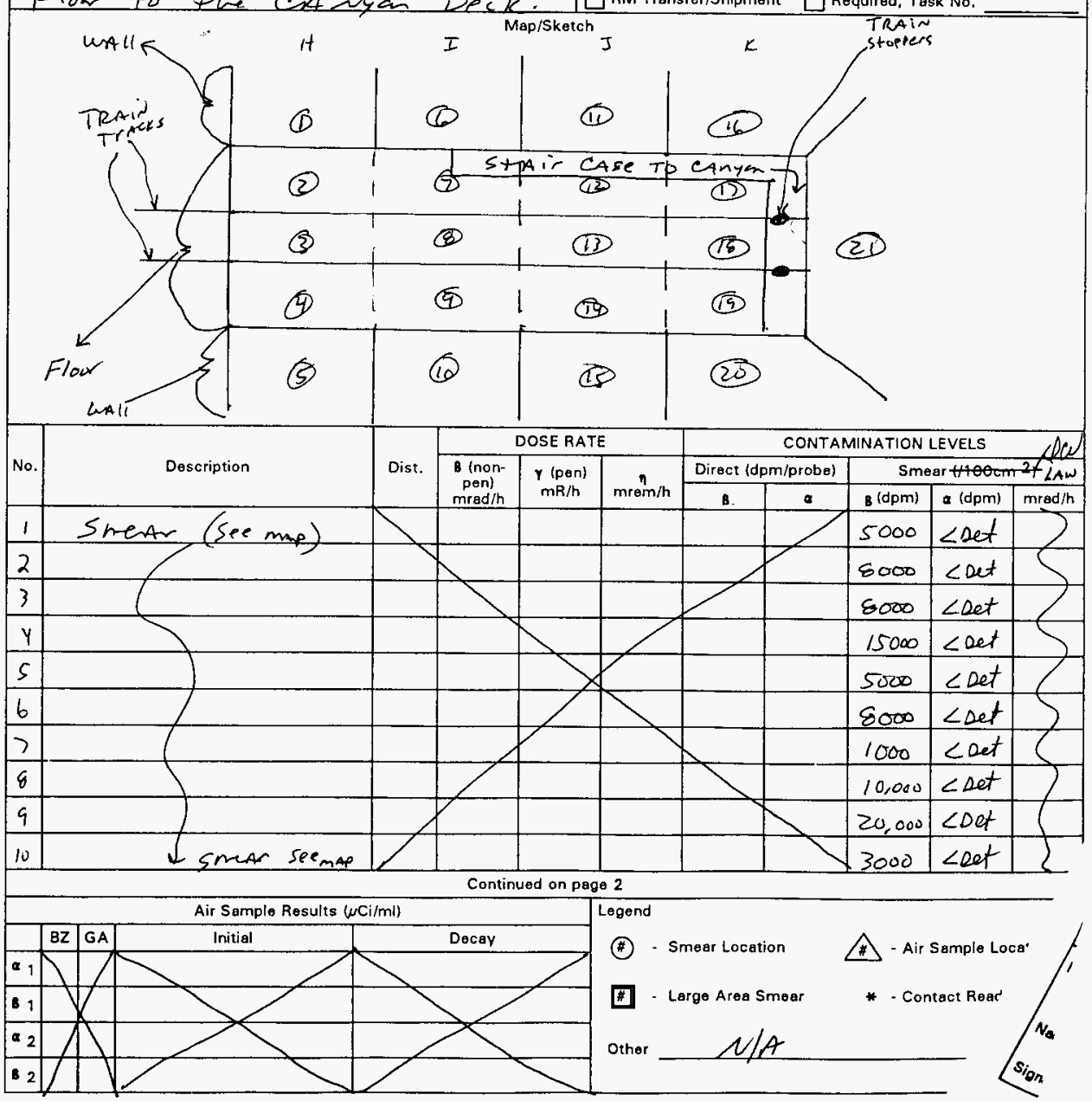

gend

(\#) - Smear Location

A

- Air Sample Loca

$\#$

Large Area Smear

* - Contact Rear

Other $N / A$ 


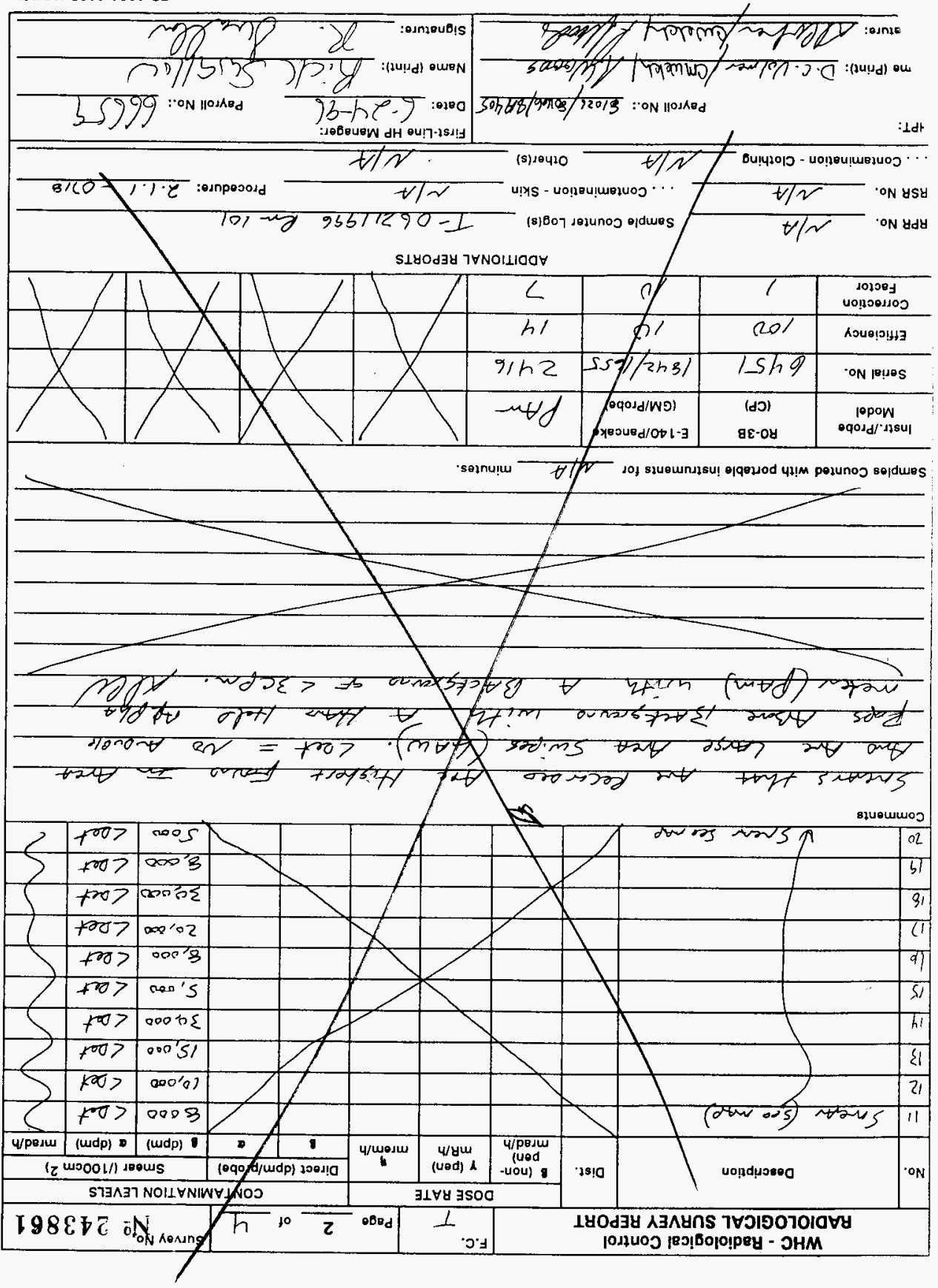


Attachment 3

Page 3 of 11

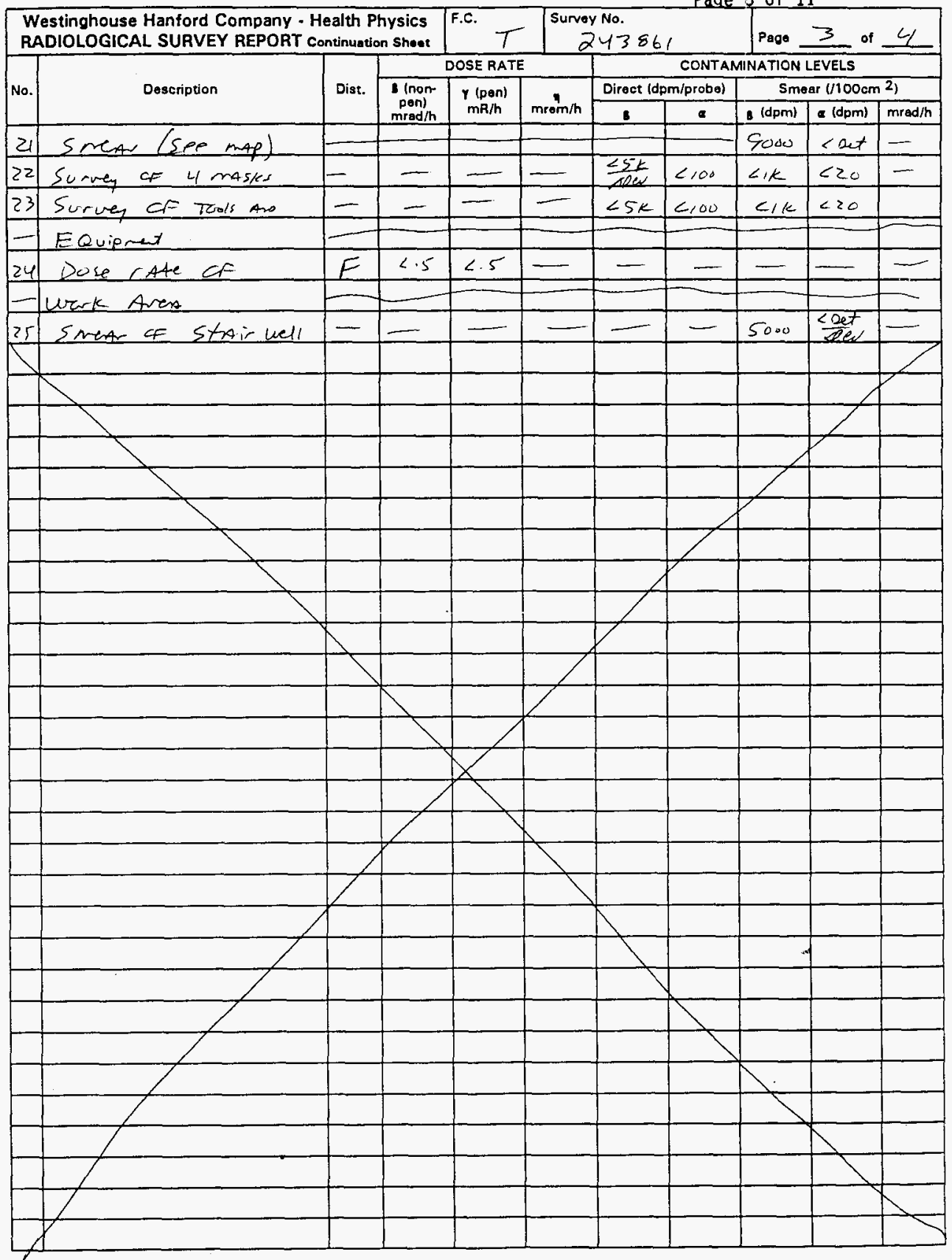




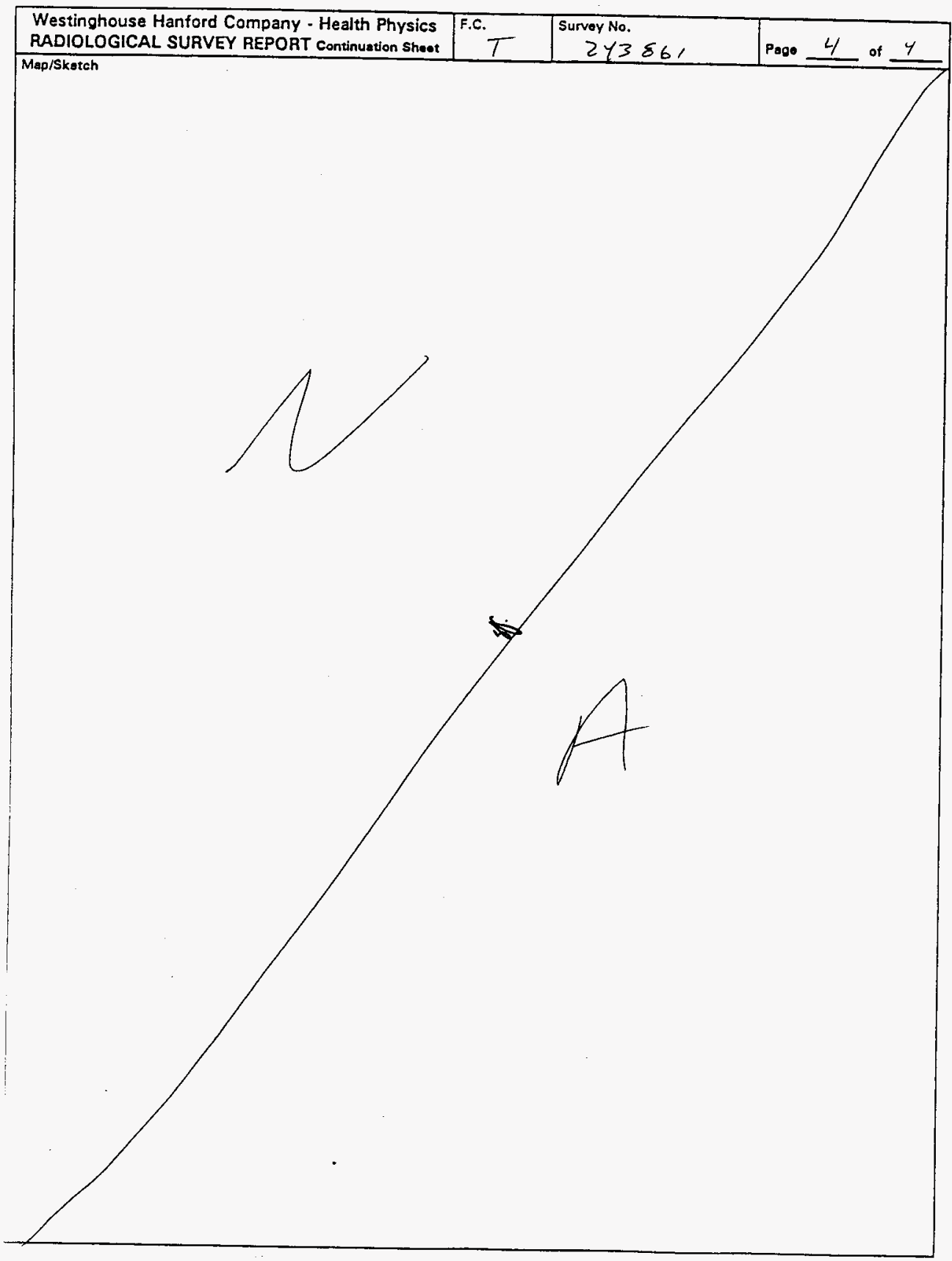

BD-6000-010.1R (01/94) 


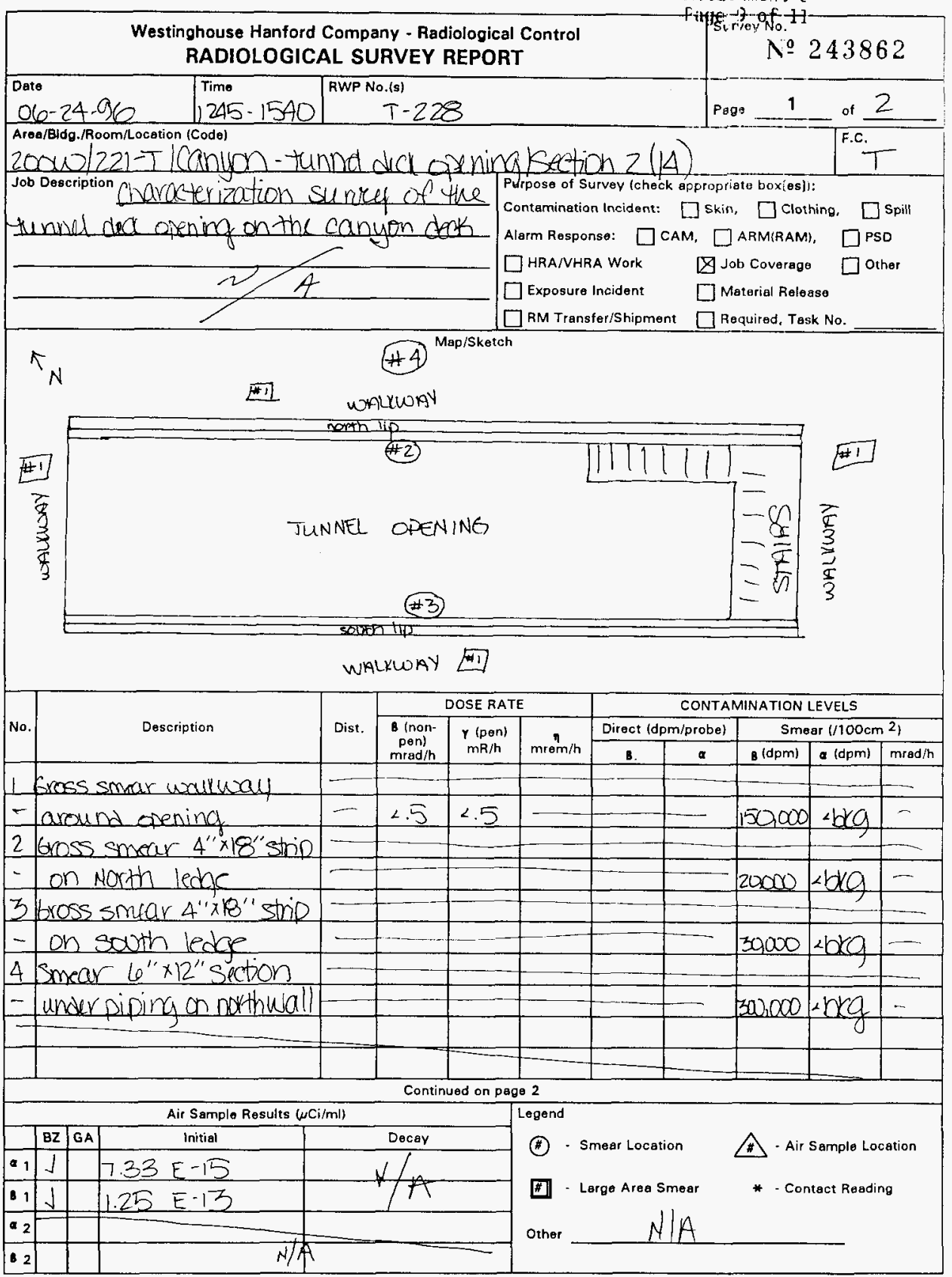




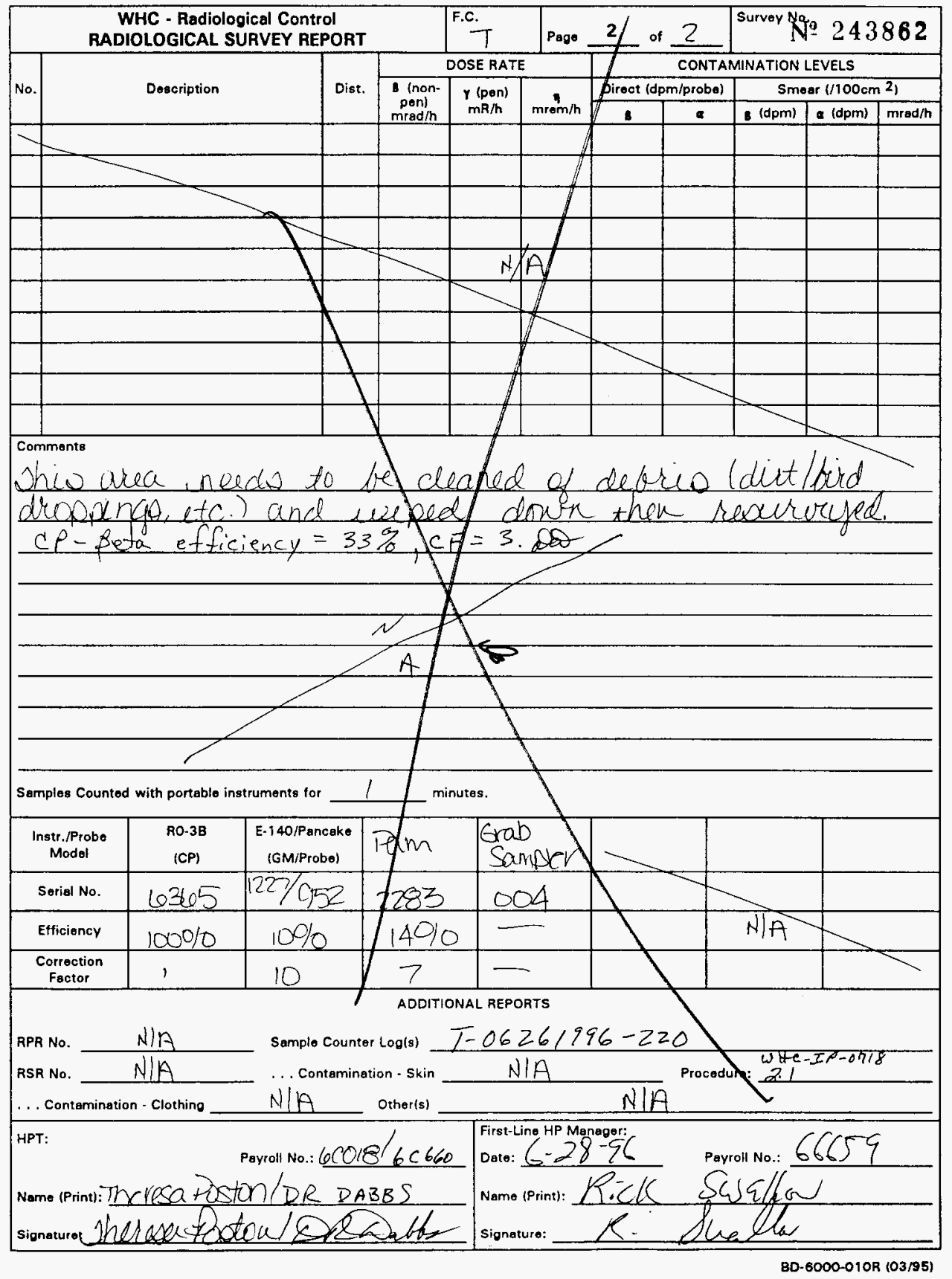


At + achment 3

Westinghouse Hanford Company - Radiological Control RADIOLOGICAL SURVEY REPORT

Fiffe-lo of 11

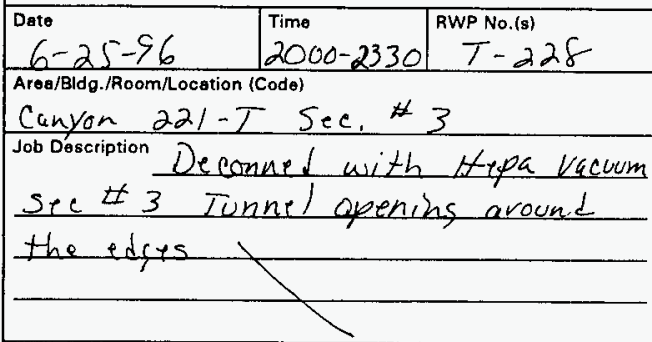

№ 243867

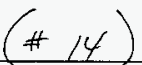

Page

$\frac{1}{\text { F.C. }}$ of

Purpose of Survey (check appropriate box[es]):

Contamination Incident: $\square$ skin, $\square$ clothing. $\square$ spill

Alerm Response: $\square$ CAM. $\square$ ARM(RAM), $\square$ PSD

$\square$ HRA/VHRA Work

$\square$ Exposure Incident

DJob Coverage $\square$ Other

[] Material Release

RM Transfer/Shipment $\square$ Required, Task No.

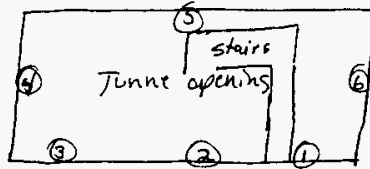

sec. $\# 4$

sec. $* 5$

No.

Description

1 General Area (working)

\begin{tabular}{|c|c|c|c|}
\hline \multirow[b]{2}{*}{$\begin{array}{r}\text { Dist } \\
(-\lambda)\end{array}$} & \multicolumn{3}{|c|}{ DOSE RATE } \\
\hline & $\begin{array}{c}\text { B (non- } \\
\text { pen) } \\
\text { mrad } / \mathrm{h}\end{array}$ & $\begin{array}{c}Y \text { (pen) } \\
m R / h\end{array}$ & $\mathrm{mrem} / \mathrm{h}$ \\
\hline
\end{tabular}

2. (1) smeconedse of opening

3 (2) smearon rdge atopening

4 (3) smeuron ctce of gorning

5 (4) Smear on edfe of mening

6. (5) Smear on tder of opening

7 (6) smear on zlce of openis

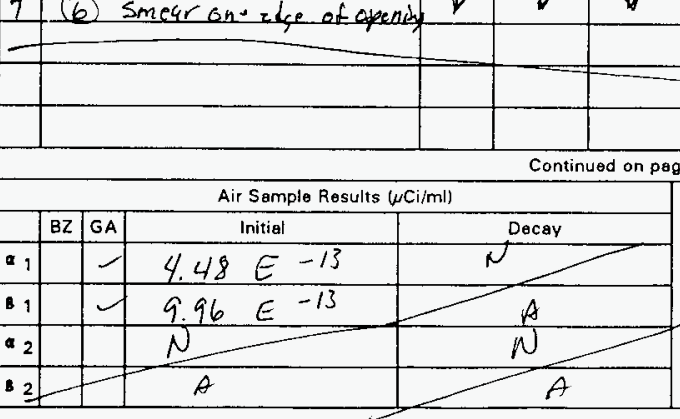

$N / S$

$N / / 2 N / A$

$|x| A$

CONTAMINATION LEVELS

Direct (dpm/probe) $\quad$ Smear $(1 / 100 \mathrm{~cm} 2$ )

\begin{tabular}{c|c|c|c|c|} 
B. & a & B (dpm) & a (dpm) & $\mathrm{mrad} / \mathrm{h}$ \\
\hline
\end{tabular}

\begin{tabular}{l|l|l|l|l|l}
$N / A$ & $N / A$ & $N / A$ & $N / A$ & $\alpha / A$
\end{tabular}

\begin{tabular}{l|l|l|l}
$N / A$ & $N / A$ & $2 K$ & 20 \\
\hline
\end{tabular}

$2 K \quad<D$

$2 k$

$1.5 K \quad<p$

$2 k$

$\angle D$




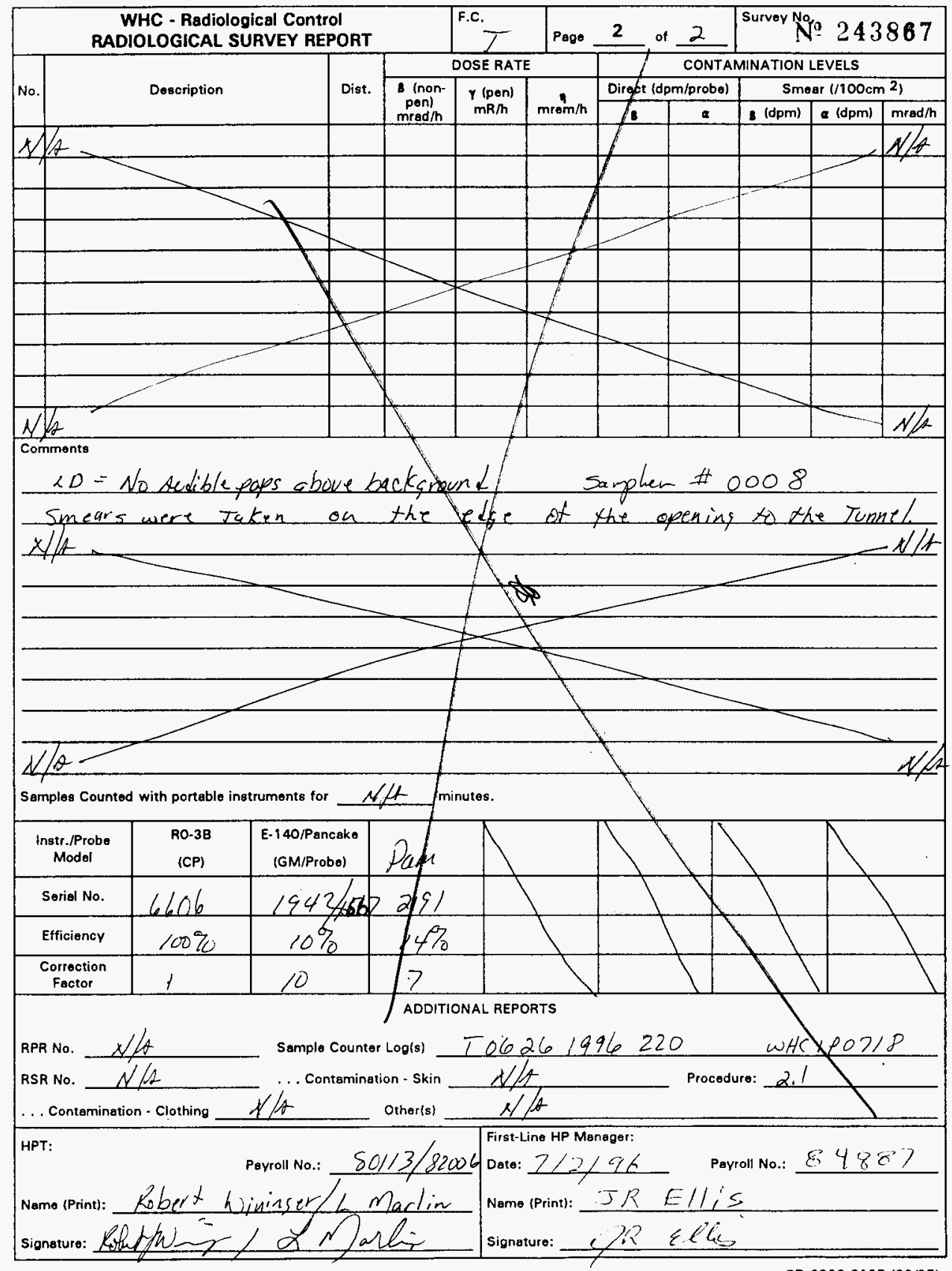


Attaciment 3

Paqe 11 of 11

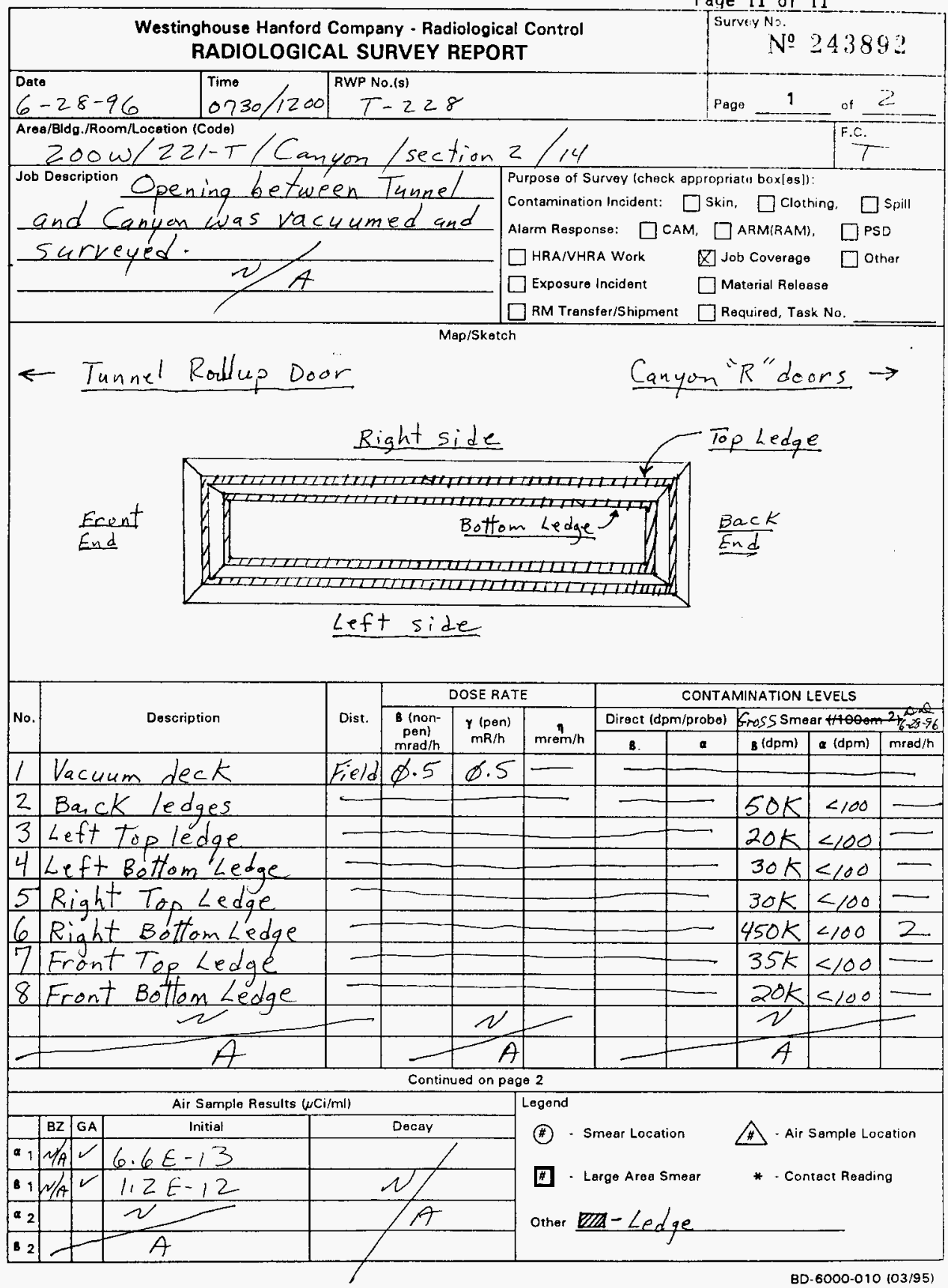




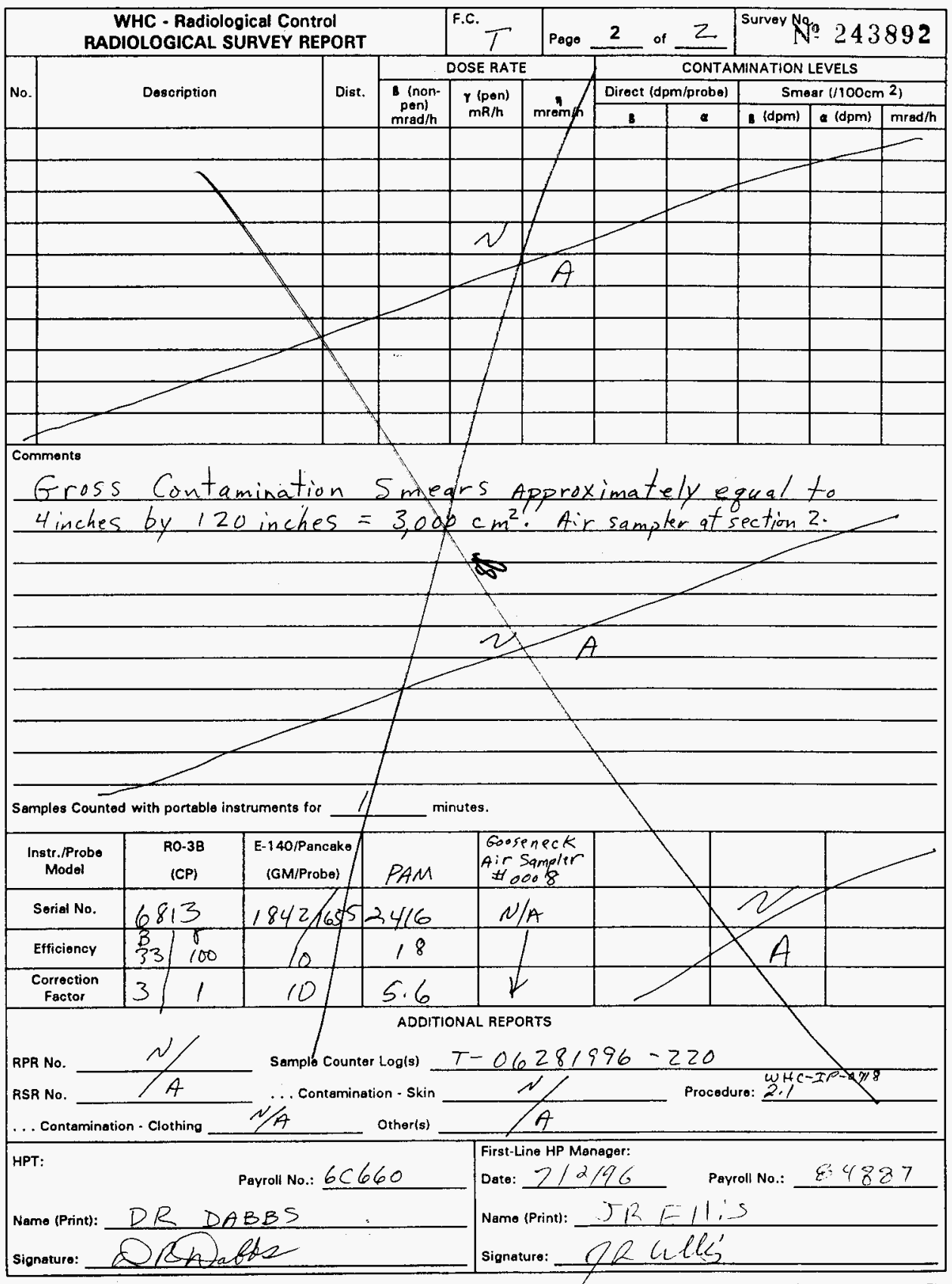


From: T Plant Radiological Control

Phone: $376-5696$ T3-20

33541-96-RBS-002

Date: April 23, 1996

Subject: AIR FLOW PATTERNS IN THE 221-T TUNNEL

$\begin{array}{ll}\text { To: } & \text { T3-28 } \\ \text { R. B. Blackford } & \text { T3-20 } \\ \text { cC: W. S. Ayers } & \text { T3-28 } \\ & \\ \text { E. J. Adams } & \text { T4-06 } \\ \text { T. L. Erickson } & \text { T3-28 } \\ \text { M. R. Fishburn } & \text { T4-06 } \\ \text { C. K. Girres } & \text { T3-28 } \\ \text { S. H. Norton } & \text { T3-28 } \\ \text { W. Smith } & \text { T3-20 } \\ \text { L. O. Waggoner } & \text { T0-06 } \\ \text { M. S. Wright } & \text { T3-28 } \\ \text { SWDPC SAT 3 } & \text { T3-28 } \\ \text { RBS File/LB } & \end{array}$

On Wednesday April 17, 1996, T Plant Radiological Control conducted an air flow pattern study to determine the direction of air movement within the tunnel and between the tunnel and canyon areas. These areas are currently airborne radioactivity areas. The purpose of the test was to verify directional flow from the tunnel to the canyon in support of an effort to remove the tunnel from airborne status. Smoke was generated at various locations within the tunnel: at approximately 100 feet from the canyon entrance at floor and head level, directly below the canyon entrance at floor and head level, and at the entrance to the canyon at the tunnel ceiting level.

The tunnel has no inherent ventilation system. The canyon ventilation system pulls air into the tunnel through openings around the manway and roll-up doors, and then into the canyon through the tunnel/canyon entry. The smoke released in the tunnel demonstrated the following characteristics:

At 100 feet from the canyon entrance, the smoke traveled towards the roll-up door at floor and head level. As the smoke rose, it hovered at approximately 10 feet, and then moved towards the canyon entrance at levels above approximately 15 feet. These height measurements are estimated.

Directly below the canyon entrance, the smoke expanded and hovered, drifting slowly upward. This upward movement was more defined when the manway door was open. At the maintenance platform level, the smoke filled the airspace, drifting upward.

At the entrance to the canyon at the tunnel ceiling level, the smoke moved directly out of the tunnel and into the canyon. The smoke moved approximately 10 feet in 10 seconds $(-1 \mathrm{ft} / \mathrm{sec})$ with the tunnel doors closed. This velocity was estimated. With the manway door open, the 
smoke rose at a faster rate, approximately 10 feet in 7 to 8 seconds $(-1.3$ to $1.4 \mathrm{ft} / \mathrm{sec})$. Once the smoke was inside the canyon, it expanded and directional flow was not discernable. After the smoke release was completed (approximately 30 minutes) a build-up of smoke could be seen over sections three and four in the north end of the canyon.

The attached drawing shows the air flow patterns observed in the tunnel.

The air flow pattern test indicates that the tunnel has the necessary directional air flow to prevent suspended contamination in the canyon air from entering the tunnel. while the tunnel doors are closed and while the manway door is open. This test could be further strengthened by quantitative measurements of air flow during normal working conditions. Administrative controls (procedures, Radiation Work Permits, etc.) as well as engineering controls (decontamination of canyon deck area near tunnel entry, etc.) should be incorporated and the area downposted before the tunnel is removed from airborne status.
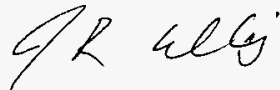

J. R. Ellis, Advanced Health Physicist

T Plant Radiological Control

jre

Attachment 
1 Remote High level Alarm for Sump 5-8 ... . . . . . . . . . . . 2

2 Remote High/High Alarm for Tank 5-7 ................ 3

3 Remote High Level Alarm for Tank 5-7 . . . . . . . . . . . . . . . . 4

4-8 Spare ......................... . . 5

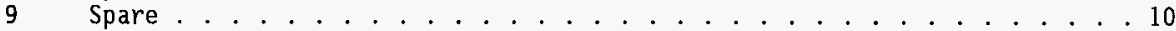

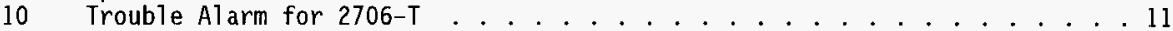

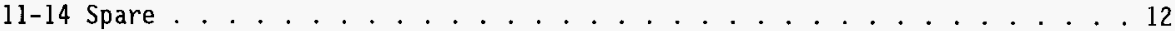

15 High Differential Pressure Alarm for the Canyon . . . . . . . . . . . 13

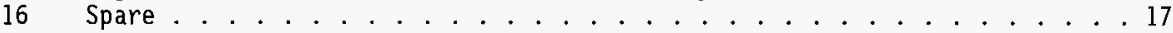

17 EVAC 2101-RMT. . . . . . . . . . . . . . . . 18

FiguRE 1 SECTION 18, ANNUNCIATOR PANEL, OPERATING GALLERY . . . . . . 19

FIGURE 2 EVAC 2101 -RMT, CANYON EVACUATION ALARM, ROOM $218 \ldots \ldots . \ldots 20$ 
FACILITY: $\quad 221-\mathrm{T}$

PANEL LOCATION: Section 18 of the Operating GaTlery. See Figure 1

WHITE

SUMP 5-8

HIGH

ALARM \#: 1

ALARM CLASS: 2

SETPOINT: $10 \mathrm{in}$.

OPERATOR ACTIONS 1. ACKNOWLEDGE the alarm.

2. NOTIFY Duty Operations Manager and EET of the alarm condition and levels in Tank 5-7 and Sump 5-8.

3. CEASE all liquid accumulation into Tank 5-7.

a. STOP all liquid waste transfers (ie.-from 2706-T, to railcars, etc..).

b. CLOSE canyon water supply valves $\mathrm{W}-\mathrm{PW}-0-20-202$ and W-PW-20-262.

4. INVESTIGATE possible causes of the alarm.

POSSIBLE CAUSES 1. Rising 1iquid in sump as a result of Tank 5-7 leakage or overfil1.

2. Liquid spilling into the sump from the canyon deck.

3. Instrument malfunction.

OTHER INDICATIONS: $\quad 2$ Small Flashing Red Lights next to Annunciator Window Audible Alarm on Annunciator Panel

Local Annunciator \#1 in Section 5 of the Operating Gallery.

ALARM DESCRIPTION: $\quad$ The sump collects overflow liquid from Tank 5-7. If more than 10 in. of liquid is detected in the sump, an audible alarm and two small flashing red lights are activated at the Section 18 annunciator panel. A local alarm \#1 should also be activated in Section 5 of the Operating Gallery.

REFERENCES WHC-SD-CP-SAR-007, T Plant Safety Analys is Report (SAR) $\mathrm{H}-2-904$ 


\section{T PLANT ALARM RESPONSE PROCEDUfFief 22}

FACILITY: $\quad 221-\mathrm{T}$

PANEL LOCATION: Section 18 of the Operating Gallery. See Figure 1

WHITE

\section{TANK 5-7}

NEAR OVERFLOW

ALARM \#: 2

ALARM CLASS: 3

SETPOINT: $90 \%$ of Capacity

OPERATOR ACTIONS 1. ACKNOWLEDGE the alarm.

2. NOTIFY Duty Operations Manager and EET of the alarm condition and levels in Tank 5-7 and Sump 5-8.

3. CEASE al1 liquid accumulation into Tank 5-7.

a. STOP all liquid waste transfers (ie.-from 2706-T, to railcars, etc..).

b. CLOSE canyon water supply valves $W-P W-0-20-202$ and $\mathrm{W}-\mathrm{PW}-20-262$.

4. INVESTIGATE possible causes of the alarm.

POSSIBLE CAUSES 1. High liquid level in Tank 5-7.

2. Instrument malfunction.

OTHER INDICATIONS: $\quad 2$ Small Flashing Red Lights next to Annunciator Window Audible Alarm on Annunciator Panel

Local Annunciator \#2 in Section 5 of the Operating Gallery.

ALARM DESCRIPTION: $\quad$ Wastes from individual canyon cells drain into a common drain header that terminates at Tank 5-7 in the deep cell 5-R. Liquid waste from Building 2706-T is discharged into cell 6-L, from which it also drains into Tank 5-7. When Tank 5-7 reaches a 1 iquid level above $90 \%$, an audible alarm and two small flashing red lights are activated at the annunciator panel. A 1 ocal alarm (see alarm \# 29) should also be activated in Section 5 of the Operating Gallery.

REFERENCES WHC-SD-CP-SAR-007, T Plant Safety Analysis Report (SAR) $\mathrm{H}-2-88613$ 
FACILITY: $\quad 221-$ T

PANEL LOCATION: Section 18 of the Operating Gallery. See Figure 1

WHITE

\section{TANK 5-7}

OVER NORMAL LIMIT

ALARM \#: 3

ALARM CLASS: 4

SETPOINT: $70 \%$ of capacity

OPERATOR ACTIONS 1. ACKNOWLEDGE the alarm.

2. NOTIFY Duty Operations Manager of the alarm condition and level of Tank 5-7.

3. CEASE all liquid generating activities in the canyon and tunnel.

4. CEASE all transfers from 2706-T, and/or railcar transfers to Tank $5-7$.

5. INSPECT the Canyon using the video cameras.

6. IF level continues to increase after 10 minutes: TRANSFER tank contents as directed by 0perations Management.

POSSIBLE CAUSES 1. High liquid level in Tank 5-7.

2. Instrument malfunction.

3. Pipe leaking in Canyon.

OTHER INDICATIONS: $\quad 2$ Small Flashing Red Lights next to Annunciator Window.

Local Alarm \# 3 in Section 5 of the Operating Gallery.

Audible Alarm on Annunciator Panel

ALARM DESCRIPTION: Wastes from individual canyon cells drain into a common drain header that terminates at Tank 5-7 in the deep cell 5-R. Liquid waste from Building 2705-T is discharged into cell 6-L, from which it also drains into Tank 5-7. When Tank 5-7 reaches a liquid Tevel above $70 \%$, an audible alarm and two small flashing red lights are activated at the annunciator panel. A local alarm \# 3 should also be activated in Section 5 of the operating Gallery.

REFERENCES WHC-SD-CP-SAR-007, T Plant Safety Analysis Report (SAR) 
FACILITY: $\quad 221-\mathrm{T}$

PANEL LOCATION: Section 18 of the Operating Gallery. See Figure 1

ALARM \#: 4

ALARM CLASS:

SETPOINT:

OPERATOR ACTIONS

POSSIBLE CAUSES

OTHER INDICATIONS:

ALARM DESCRIPTION:

REFERENCES: 
FACILITY: $\quad 221-\mathrm{T}$

PANEL LOCATION: Section 18 of the Operating Gallery. See Figure 1

ALARM \# : 5

ALARM CLASS:

SETPOINT:

OPERATOR ACTIONS

POSSIBLE CAUSES

OTHER INDICATIONS:

ALARM DESCRIPTION:

REFERENCES: 
FACILITY : $\quad$ 221-T

PANEL LOCATION: Section 18 of the 0perating Gallery. See Figure 1

ALARM \#: 6

ALARM CLASS:

SETPOINT:

OPERATOR ACTIONS

POSSIBLE CAUSES

OTHER INDICATIONS:

ALARM DESCRIPTION:

REFERENCES: 
FACILITY: $\quad 221-T$

PANEL LOCATION: Section 18 of the Operating Gallery. See Figure 1

ALARM \#: 7

ALARM CLASS:

SETPOINT:

OPERATOR ACTIONS

POSSIBLE CAUSES

OTHER INDICATIONS:

ALARM DESCRIPTION:

REFERENCES : 


\section{FACILITY: $\quad 221-T$}

PANEL LOCATION: Section 18 of the Operating Gallery. See Figure 1

\section{SPARE}

ALARM \#: 8

ALARM CLASS:

SETPOINT:

OPERATOR ACTIONS

POSSIBLE CAUSES

OTHER INDICATIONS:

ALARM DESCRIPTION:

REFERENCES: 
FACILITY: $221-T$

PANEL LOCATION: Section 18 of the Operating Gallery. See Figure 1

WHITE

Spare

ALARM \#: 9

ALARM CLASS:

SETPOINT:

OPERATOR ACTIONS

POSSIBLE CAUSES

OTHER INDICATIONS

ALARM DESCRIPTION

REFERENCES 


\section{T PLANT ALARM RESPONSE PROCEDURE 13 of 22}

FACILITY: $\quad 221-\mathrm{T}$

PANEL LOCATION: Section 18 of the 0perating Gallery. See Figure 1

WHITE

\section{$2706 \mathrm{~T}$ TROUBLE}

ALARM \# : 10

ALARM CLASS: 4

SETPOINT: $\quad \mathrm{N} / \mathrm{A}$

OPERATOR ACTIONS 1. ACKNOWLEDGE the alarm.

2. NOTIFY Duty Operations Manager of the alarm condition.

3. DETERMINE which of the possible causes has initiated and take appropriate alarm response actions (see ARP-D-463-00001).

POSSIBLE CAUSES

1. 2706-T master alarm switched off for ACT-1.

2. Any alarm associated with the 2706-T alarm panel is activated.

3. Normal shutdown of the 2706-T ventilation system.

4. Instrument malfunction.

5. Power failure at 2706-T.

OTHER INDICATIONS: $\quad 2$ Small Flashing Red Lights next to Annunciator Window Audible Alarm on Annunciator Panel

ALARM DESCRIPTION: $\quad$ The Trouble Alarm is activated when any alarm at the 2706-T building is actuated (see ARP-D-463-00001, 2706-T Alarm Response Procedure for descriptions). Such an occurrence is indicated by two small flashing red lights next to the white annunciator window and an audible alarm on the annunciator panel. This alarm will also be activated when the 2706 ACT-1 ventilation system master alarm switch is shut off.

REFERENCES $\mathrm{H}-2-83100, \mathrm{H}-2-83103$ 
FACILITY: $\quad 221-T$

PANEL LOCATION: Section 18 of the Operating Gallery. See Figure 1

SPARE

ALARM \#: 11

ALARM CLASS:

SETPOINT:

OPERATOR ACTIONS

POSSIBLE CAUSES

OTHER INDICATIONS:

ALARM DESCRIPTION:

REFERENCES: 


\section{T PLANT ALARM RESPONSE PROCEDUFIE}

FACILITY: $221-T$

PANEL LOCATION: Section 18 of the Operating Gallery. See Figure 1

SPARE

ALARM \#: 12

ALARM CLASS:

SETPOINT:

OPERATOR ACTIONS

POSSIBLE CAUSES

OTHER INDICATIONS:

ALARM DESCRIPTION:

REFERENCES : 
FACILITY : $\quad 221-\mathrm{T}$

PANEL LOCATION: Section 18 of the Operating Gallery. See Figure 1

ALARM \#: 13

ALARM CLASS:

SETPOINT:

OPERATOR ACTIONS

POSSIBLE CAUSES

OTHER INDICATIONS:

ALARM DESCRIPTION:

REFERENCES : 
FACILITY: $\quad 221-\mathrm{T}$

PANEL LOCATION: Section 18 of the Operating Gallery. See Figure 1

SPARE

ALARM \#: 14

ALARM CLASS:

SETPOINT :

OPERATOR ACTIONS

POSSIBLE CAUSES

OTHER INDICATIONS:

ALARM DESCRIPTION:

REFERENCES : 
FACILITY: $\quad 221-T$

PANEL LOCATION: Section 18 of the Operating Gallery. See Figure 1

WHITE

CANYON

HIGH DP

ALARM \#: 15

ALARM CLASS: 3

SETPOINT: -0.70 in. $w . g$.

OPERATOR ACTIONS 1. ACKNOWLEDGE the alarm.

2. NOTIFY Duty Operations Manager of the alarm condition (Duty Ops.Manager will determine corrective actions).

3. IF the alarm does NOT clear within 5 minutes:

a. EVACUATE personnel in the Canyon and Crane Cab.

b. ILLUMINATE Canyon DO NOT ENTER sign using switch in Rm. 218.

c. CHECK for abnormal differential pressures of the operating exhaust filter banks.

NOTE: Maximum DP allowed by the SAR is 1.00 in. w.g.

POSSIBLE CAUSES 1. Filter failure.

2. Supply fan malfunction.

3. Instrument malfunction.

OTHER INDICATIONS: $\quad 2$ Small Flashing Red Lights next to Annunciator Window Audible Alarm on Annunciator Panel

ALARM DESCRIPTION: $\quad$ Failure to maintain a proper differential pressure (DP) could endanger the Plant structure and stability (especially the canyon doors). When DP exceeds 0.70 in. w.g., an audible alarm sounds and two small red lights flash next to the window at the annunciator panel

REFERENCES WHC-SD-CP-SAR-007, T Plant Safety Analysis Report (SAR) 
FACILITY : $\quad 221-T$

PANEL LOCATION: Section 18 of the Operating Gallery. See Figure 1

ALARM \#: 16

ALARM CLASS :

SETPOINT:

OPERATOR ACTIONS

POSSIBLE CAUSES

OTHER INDICATIONS:

ALARM DESCRIPTION:

REFERENCES: 


\section{FACILITY: $\quad 221-T$}

PANEL LOCATION: Section 18 of the 0perating Gallery. See Figure 2

ALARM \#: 17 (EVAC 2101-RMT)

ALARM CLASS: 2

SETPOINT: -0.06 in. w.g.

OPERATOR ACTIONS 1. NOTIFY Duty Operations Manager of the alarm condition (Duty Ops. Manager will determine corrective actions).

2. STOP decontamination activities.

3. IF the cause of the canyon low DP alarm is due to opening the tunnel roll-up door, operations will be limited in order to control the spread of contamination.

4. IF the cause of the low DP alarm is NOT due to the opening of the roll-up door, and the DP cannot be restored within 5 minutes:

a. EVACUATE personnel in the canyon, tunnel and crane cab.

b. ILLUMINATE canyon "DO NOT ENTER" sign using the switch in Room 218.

NOTE - The following is an $L C O$ as required by the SAR.

NOTE: The SAR does not allow decontamination work in the canyon if the DP is below $0.025 \mathrm{in}$. w.g. Also, the SAR states that the minimum allowable alarm set point is 0.030 in. w.g.

POSSIBLE CAUSES 1. Gusty winds, causing a temporary fluctuation in Canyon pressure.

2. Tunnel door open.

3. Supply fan malfunction.

4. Loss of exhaust fan.

5. Instrument malfunction.

OTHER INDICATIONS:

ALARM DESCRIPTION:
- $\quad$ Audible alarm in Room 218.

- If alarm is silenced, "TROUBLE" LED will illuminate.

- Horn and strobe in canyon and tunnel.

Failure to maintain an adequate differential pressure (DP) between the canyon and the operating Gallery could result in the release of radionuclides from the $T \mathrm{Pl}$ ant Canyon. The negative pressure in the canyon with respect to the Operating Gallery is required to prevent airborne contamination from moving to the occupied areas of the building or to the environment. When DP decreases to -0.06 in. w.g. or less, an audible alarm sounds and strobes flash in the canyon and tunnel. Duty Operations Supervisor can silence alarm tone (slow whoop) by activating the tone silence switch. (See Figure 2) The white strobe light will continue to flash until the $D P$ returns to normal.

REFERENCES WHC-SD-CP-SAR-007, T Plant Safety Analysis Report (SAR); 0SD-D-187-00008, Section 1.8.1 


\section{T PLANT ALARM RESPONSE PROCEDUAEE 21 of 22}

\begin{tabular}{|c|c|c|c|c|}
\hline $\begin{array}{c}\text { SUMP 5-8 } \\
\text { HIGH }\end{array}$ & $\begin{array}{l}0 \\
0\end{array}$ & $\begin{array}{c}\text { PMR } \\
0 \\
\text { ON }\end{array}$ & $\begin{array}{l}0 \\
0\end{array}$ & SPARE \\
\hline $\begin{array}{l}\text { TANMK 5-7 } \\
\text { NEAR } \\
\text { OVERFLON }\end{array}$ & 0 & & 0 & $\begin{array}{c}2706-T \\
\text { TROUBLE }\end{array}$ \\
\hline $\begin{array}{l}\text { TANK 5-7 OVER } \\
\text { NORMALLL LIMIT }\end{array}$ & 0 & & 0 & SPARE \\
\hline SPARE & 0 & & 0 & SPARE \\
\hline SPARE & 0 & & 0 & SPARE \\
\hline SPARE & 0 & & 0 & SPARE \\
\hline SPARE & $\begin{array}{l}0 \\
0\end{array}$ & & 0 & $\begin{array}{l}\text { CANYON } \\
\text { HIGH DP }\end{array}$ \\
\hline SPARE & 0 & & 0 & SPARE \\
\hline TEST & SL & & ACK & SET \\
\hline
\end{tabular}
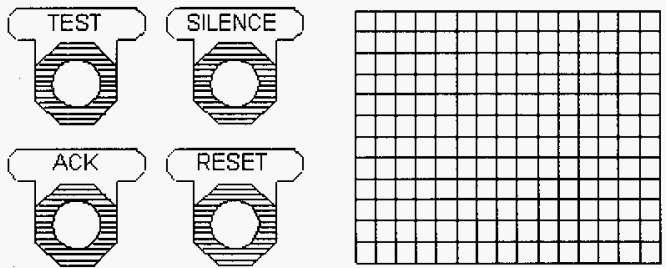

SECTION 18 ANNWUNCIATOR PANEL OPERATING GALLERY

FIGURE 1 - SECTION 18, ANNUNCIATOR PANEL, OPERATING GALLERY 


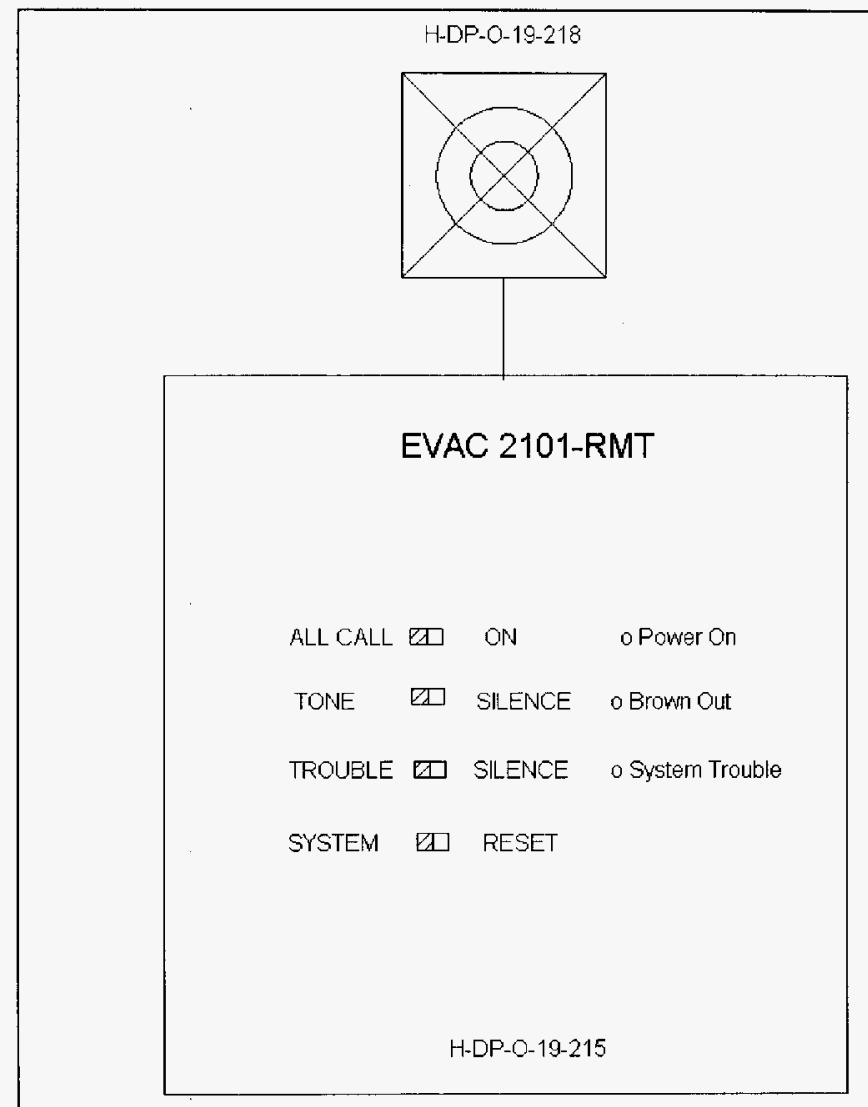

FIGURE 2 - EVAC 2101-RMT Evacuation Alarm, Room 218 